\title{
Interaction of weak shock waves with cylindrical and spherical gas inhomogeneities
}

\author{
By J.-F. HAAS $†$ AND B. STURTEVANT \\ Graduate Aeronautical Laboratories, California Institute of Technology, \\ Pasadena, CA 91125, USA
}

(Received 26 February 1986 and in revised form 10 December 1986)

The interaction of a plane weak shock wave with a single discrete gaseous inhomogeneity is studied as a model of the mechanisms by which finite-amplitude waves in random media generate turbulence and intensify mixing. The experiments are treated as an example of the shock-induced Rayleigh-Taylor instability, or RichtmyerMeshkov instability, with large initial distortions of the gas interfaces. The inhomogeneities are made by filling large soap bubbles and cylindrical refraction cells $(5 \mathrm{~cm}$ diameter) whose walls are thin plastic membranes with gases both lighter and heavier than the ambient air in a square $(8.9 \mathrm{~cm}$ side shock-tube text section. The wavefront geometry and the deformation of the gas volume are visualized by shadowgraph photography. Wave configurations predicted by geometrical acoustics, including the effects of refraction, reflection and diffraction, are compared to the observations. Departures from the predictions of acoustic theory are discussed in terms of gasdynamic nonlinearity. The pressure field on the axis of symmetry downstream of the inhomogeneity is measured by piezoelectric pressure transducers. In the case of a cylindrical or spherical volume filled with heavy low-sound-speed gas the wave which passes through the interior focuses just behind the cylinder. On the other hand, the wave which passes through the light high-sound-speed volume strongly diverges. Visualization of the wavefronts reflected from and diffracted around the inhomogeneities exhibit many features known in optical and acoustic scattering. RayleighTaylor instability induced by shock acceleration deforms the initially circular cross-section of the volume. In the case of the high-sound-speed sphere, a strong vortex ring forms and separates from the main volume of gas. Measurements of the wave and gas-interface velocities are compared to values calculated for onedimensional interactions and for a simple model of shock-induced Rayleigh-Taylor instability. The circulation and Reynolds number of the vortical structures are calculated from the measured velocities by modeling a piston vortex generator. The results of the flow visualization are also compared with contemporary numerical simulations.

\section{Introduction}

The interaction of shock waves with fluid non-uniformities modifies the geometry and amplitude of the wave fronts by reflection, refraction, diffraction and scattering, and modifies the morphology of the inhomogeneities by fluid deformation, vorticity and entropy production, and transport. The interaction of shocks with non-uniform

$\dagger$ Present address: Commissariat a l'Energie Atomique, Centre d'Etudes de Limeil-Valenton, BP27, 94190 Villeneuve Saint Georges, France. 
media occurs often in science and technology, for example, sonic boom propagation through the Earth's turbulent atmosphere (Ffowes Williams \& Howe 1973) shock boundary-layer interaction on transonic airfoils (Anyiwo \& Bushnell 1982) and shock wave interactions with unstable interfaces between thermonuclear fuel and outer shell material of laser fusion pellets (Andronov et al. 1979). Clearly, the description of such interactions is complicated and simple analytical models are difficult to formulate. As a consequence, it is necessary to carry out exploratory experiments to elucidate the important processes involved. The simple gas inhomogeneities considered in the present study can be thought of as the building blocks of more complicated inhomogeneous media. We limit our attention to discrete inhomogeneities because the flow visualization is especially graphic. It is easy to arrange very large index-of-refraction variations in acoustic media; in the present experiments, with a weak shock wave propagating from atmospheric air into helium or Refrigerant 22 (R22), the acoustic index of refraction takes on the values 0.35 and 1.88 , while the ratios of acoustic impedances are 0.41 and 1.65 , respectively. Therefore, the inhomogeneities are very strong acoustic lenses, and focal lengths are of the order of the diameter of the inhomogeneities.

\subsection{Wave effects}

In the interaction of a shock wave with a spherical or cylindrical volume of gas of different density and/or sound speed, wave reflection refraction, diffraction and focusing are important. The refraction of shock waves at plane gas interfaces has been examined by Jahn (1956), Abd-el-Fattah, Henderson \& Lozzi (1976), Abd-el-Fattah \& Henderson $1978 a, b)$ and Catherasoo \& Sturtevant (1983). Depending on the angle of incidence of the shock wave onto the interface and on the strength of the shock, the refraction can be regular (incident, reflected and refracted waves intersect the interface at the same point) or irregular (the refracted shock intersects the interface ahead of the incident shock). Furthermore, in the case of the so-called slow-fast interface, for which the gas downstream of the interface has a higher sound speed than the gas upstream, the transmitted wave can run ahead of the first disturbance in the slow medium, leading to a 'precursor' configuration. The refraction of a shock from cylindrical or spherical interfaces covers the complete range of angles of incidence and, therefore, of all types of refraction. In view of the complexity of the plane refraction problem, it is not surprising that little attention has been given to the interaction of plane shock waves with curved gas interfaces. In a related problem, Markstein $(1957 a, b)$ and Rudinger (1958) studied the interaction of shock waves with curved flame fronts, and so considered the curved slow-fast case. Precursors and lateral shocks associated with the phenomenon of irregular refraction were identified.

As described in $\$ 2$, the extensive literature on the refraction, reflection and diffraction of waves of infinitesimal amplitude (e.g. Pierce 1981; Friedlander 1958), provides a useful basis for considering the distortion of weak shocks by fluid inhomogeneities that act as lenses. Even relatively rarely observed effects such as tunnelling or glory (Jones 1978; Marston \& Kingsbury 1981; Marston \& Langley 1983) have analogues in the nonlinear case. In the field of ultrasonics, considerable research has been carried out on the properties of cylindrical and spherical sonar targets. The geometry of the transmitted waves in the case of liquid-filled cylinders (Brill \& Überall 1970) and in the case of metal cylinders (Neubauer \& Dragonette 1970) and of the reflected waves (Folds 1971) has been identified. The configuration of the internal refracted and reflected waves has been observed in the case of 
mechanical impact on liquid-filled cylindrical containers (Bockhoff \& Rauch 1973). The analogous phenomena of light wave interaction with spherical particles are well documented (e.g. Van de Hulst 1957).

\subsection{Distortion of the volume and mixing}

As the shock sweeps over the inhomogeneity, the shape of the gas volume changes due to compression and the differential motions induced. In the simplest case, when the substance in the volume is not different from that outside, a shape of circular cross-section with diameter $D$ deforms into an ellipse of major axis $D$ and minor axis $D\left(1-V_{2} / V_{s}\right)$ where $V_{s}$ and $V_{2}$ are the velocities of the shock and the gas behind the shock. In the case of different fluids, normal interaction on the axis of symmetry again leads to compression, while oblique and tangential off-axis interactions lead, in addition, to shear (Chu \& Kovasznay 1957). Vorticity is produced because of the misalignment of the gradients in pressure and density (or entropy), as shown by the vorticity production equation.

$$
\frac{\mathrm{d} \omega}{\mathrm{d} t}=(\omega \cdot \nabla) \mathrm{V}-\omega \boldsymbol{\nabla} \cdot \mathbf{V}+\frac{\boldsymbol{\nabla} \rho \times \nabla p}{\rho^{2}},
$$

where $\omega$ is the vorticity, $\mathrm{V}$ the velocity, $p$ the pressure, and $\rho$ the density. When a shock is incident, say from the right, on a low-density (e.g. helium) sphere, because the light gas is relatively easier to accelerate, clockwise vorticity is produced at the top and counterclockwise vorticity is generated at the bottom of the volume. Consequently, it might be expected that during the subsequent motion, because of shear-layer instability and vortex roll-up, the inhomogeneity would transform into a vortex-ring-like structure, and by vortex induction the structure would move downstream (to the left) relative to the surrounding fluid. On the other hand, for a shock incident on a high-density (e.g. R22) sphere, the sense of the vorticity is opposite, and the structure moves upstream against the stream. Thus, the expectation is that after some time a spherical inhomogeneity becomes a vortex ring, while a cylindrical inhomogeneity becomes a pair of vortex lines. This process has been the object of a previous experimental study (Rudinger \& Somers 1960) in which the velocities of the vortical structures resulting from the shock-induced acceleration of small-diameter cylindrical inhomogeneities of $\mathrm{He}$ or $\mathbf{S F}_{6}$ were measured and compared with the predictions of a simple theoretical model of vortex generation by impulsive acceleration of an imaginary plate.

The initial deformation of the inhomogeneity can also be interpreted in terms of the Rayleigh-Taylor instability of accelerated, curved interfaces separating fluids of different density (Taylor 1950). The acceleration is caused by the shock wave and takes place during the first few reverberation times $\tau$ after shock impact, where, in the present case, $\tau$ is of the order $D / a$, and $a$ is a characteristic wave speed. Thereafter the acceleration is zero, so the violent motion induced by the shock, and the consequent secondary instabilities and mixing, slowly die out by viscous dissipation. Shock-induced interfacial instability has been observed in shock wave-flame interaction experiments by Markstein (1957a, b; cf. \$1.1). Curved flame fronts, when accelerated by a shock wave, undergo heavy distortions such as shape reversal and spike formation (Markstein 1957b). The deformation histories are similar to the ones observed in the case of gas bubbles suddenly released in liquids (Walters \& Davidson 1962, 1963).

The shock-induced Rayleigh-Taylor instability of a sinusoidally perturbed plane 
interface, sometimes referred to as the Richtmyer-Meshkov instability, was treated by Richtmyer (1960), and Markstein (1957a). In the linear regime, the growth rate $v$ of interface perturbations is proportional to the product of $V$, the initial amplitude $\eta_{0}$, the wavenumber $k$ of the corrugation, and the Atwood number,

$$
A=\frac{\rho_{2}-\rho_{1}}{\rho_{2}+\rho_{1}}
$$

where $\rho_{1}$ and $\rho_{2}$ are the gas densities upstream and downstream of the interface, respectively. For highly curved interfaces and very different gases the growth rate or perturbation velocity $v$ is comparable to $V$ and can be very large. Richtmyer's (1960) numerical treatment accounted for the effects of compressibility but otherwise confirmed this relation.

The deformation under shock-induced acceleration of a sinusordally perturbed plane interface oriented normal to the direction of shock propagation has been investigated with experimental conditions rather close to the work presented here (Meshkov 1970). The lower-than-expected deformation velocities observed by Meshkov were attributed to experimental difficulties (imprecision in the measurements, gas contamination) and the neglect of some factors such as viscosity in the theoretical calculations (Meyer \& Blewett 1972). Other possible effects such as drag force on the spike, and turbulence have also been mentioned (Baker \& Freeman 1981).

While this experimental investigation was in progress, a computer simulation of Markstein's shock wave-spherical flame interaction experiment was made by integrating the classical conservation equations, and the vorticity production equation (1) was analytically integrated (Picone et al. 1984). The same approach has been used to simulate the experiments described here (Picone \& Boris 1985, 1986; Picone et al. 1986).

\section{Acoustic description of the wave processes}

To a first approximation, the wavefronts generated by the interaction of a weak plane shock wave with a cylindrical or spherical volume can be exhibited and classified by ray tracing and geometrical acoustics. Then the function of experiments in which finite-amplitude waves occur is to elucidate the effects of nonlinear propagation and volume deformation. The effect of the perturbing gas is that of an acoustic 'lens' of index of refraction.

$$
n=\frac{a_{1}}{a_{2}}
$$

where $a_{1}$ and $a_{2}$ are the sound speeds of the air outside and of the gas inside, respectively. The rays of the acoustic wavefronts are straight lines in regions of constant sound speed, and refract at the boundaries of such regions according to Snell's law,

$$
\sin \theta_{\mathbf{i}}=n \sin \theta_{\mathbf{r}},
$$

where $\theta_{i}$ and $\theta_{\mathrm{r}}$ are the angles of the incident and refracted rays, respectively. The rays reflect at the boundaries such that the angle of reflection is equal to the angle of incidence.

For clarity, only the rays arising from the interaction of the incident wave with the top half of the volume are shown in the figures of this section. The incident wave 


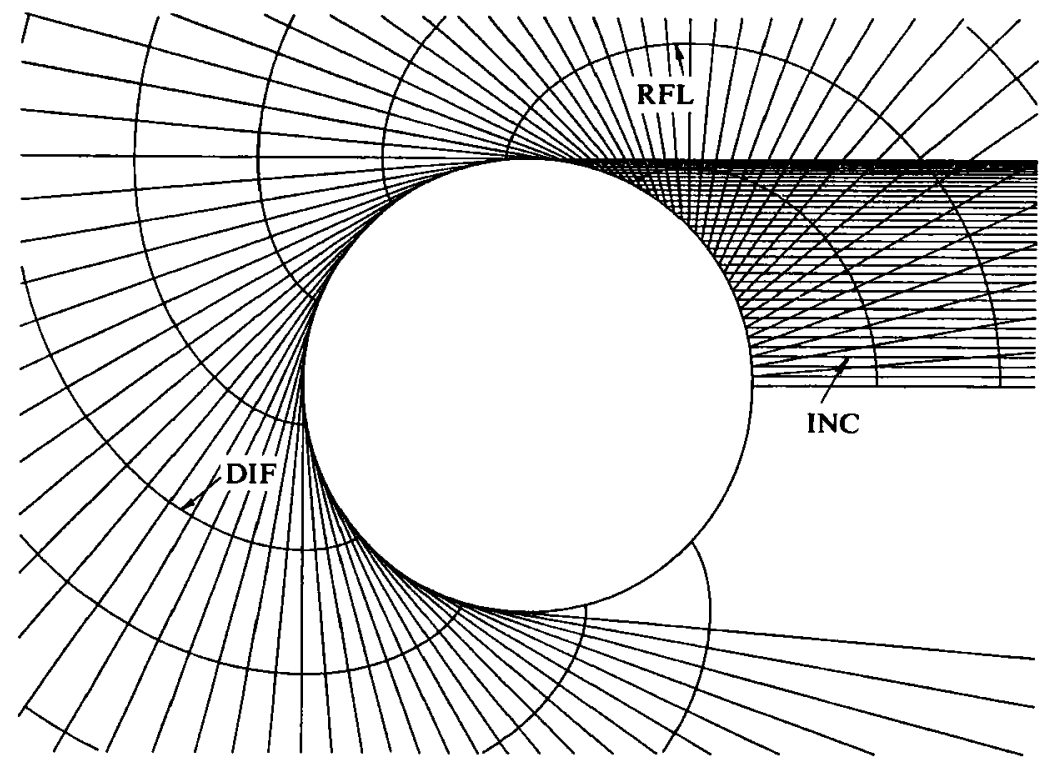

Figure 1. External reflected and diffracted rays and wavefronts: INC, incident; RFL, reflected; DIF, diffracted.

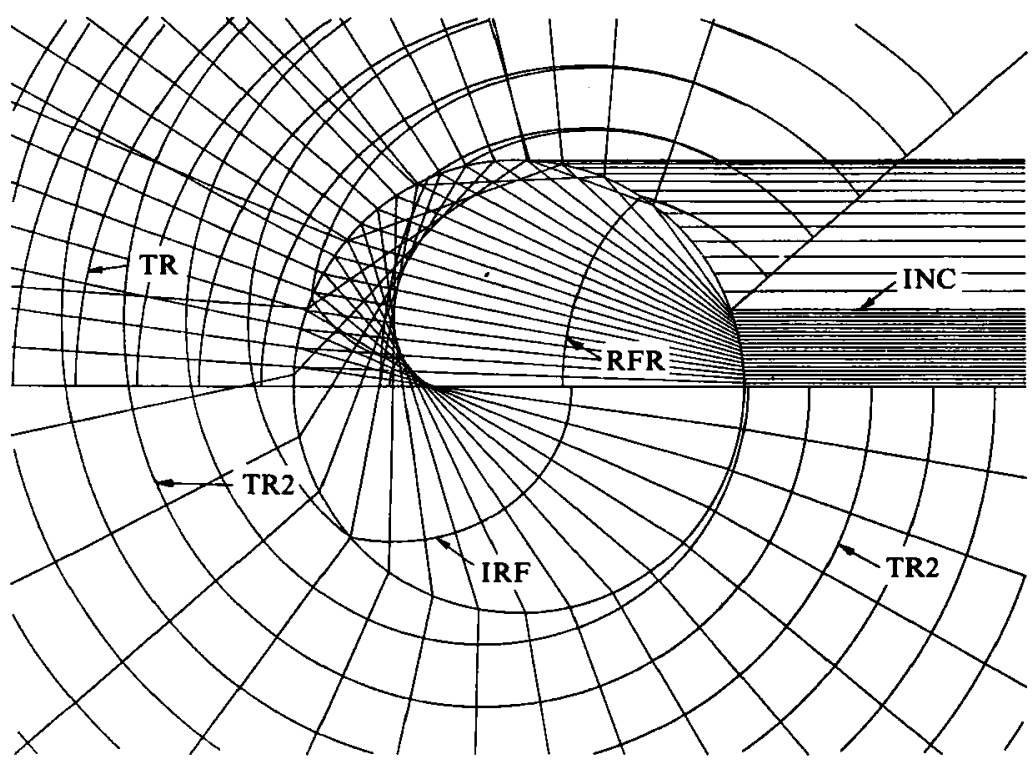

FigURE 2. Rays and wavefronts characteristic of the divergent case: RFR, refracted; IRF, internally reflected; TR, transmitted; TR2, secondary transmitted.

is represented by a family of parallel rays incident from the right onto the circular boundary at angles of incidence increasing by steps of $5^{\circ}$, except in figure 2 , in which the steps are $1^{\circ}$. The spacing between the wavefronts is chosen to correspond to a time interval of $40 \mu \mathrm{s}$ for shock interaction with a $50 \mathrm{~mm}$ diameter cylinder, except in figure 2 , in which the steps are $20 \mu \mathrm{s}$.

Figure 1 illustrates the external reflected and diffracted rays and wavefronts. These 
waves are common to all cases and will not be redrawn on the subsequent figures. The grazing ray at the top of the cylinder defines the boundary of the shadow region behind the cylinder. According to the geometrical theory of diffraction (Keller 1955, 1958), the wave diffracted into the shadow region springs from a curved diffracted ray on the boundary which sheds straight diffracted rays tangentially into the shadow region. The amplitude of the surface diffracted wave, initially some fraction of the incident amplitude dependent upon the material properties and the geometry of the problem, decreases exponentially as it propagates along the surface. The amplitude of the tangentially shed diffracted waves is, in turn, some fraction of the local surface wave amplitude.

\subsection{Divergent case}

The helium-filled cylinders and spheres are strongly divergent lenses, but only rays with $\theta_{i}$ less than the critical angle, $19^{\circ}$, are refracted inside. All the rays shown on figure 2 originate from this ray tube. At the downstream boundary they again refract and form the external wave to which we refer as the transmitted wave, and they reflect and reverberate inside the volume. The internal singly-reflected rays form a caustic that begins at the critically-refracted incident ray and loops downstream all the way to the axis of symmetry. After passing through the caustic the internal rays reflect again from the interface, forming secondary reflected waves inside and emerging outside as secondary transmitted waves. As this process repeats indefinitely, the waves rapidly weaken. In the case of a spherical lens, the forward-scattered secondary transmitted rays seem to originate from a ring-like source within the lens and they focus on the axis of symmetry; in the optics literature they are known as forward glory (Van de Hulst 1957).

Both the primary and the secondary transmitted wavefronts are tangent to the reflected wave (shown on figure 1 at the upper right) along the reflected ray at the critical angle. Both families of waves (also identified by Brill \& Überall 1970) appear much earlier than the diffracted wave of figure 1 because of the high internal wave speed.

\subsection{Convergent case}

$\mathrm{R} 22$ is a fluorocarbon $\left(\mathrm{CHClF}_{2}\right)$ with a vapour density of $3.69 \mathrm{~kg} / \mathrm{m}^{3}$ and a speed of sound of $184 \mathrm{~m} / \mathrm{s}$ at atmospheric pressure and $25^{\circ} \mathrm{C}$. The volume filled with $\mathrm{R} 22$ is a strongly convergent lens. Figure 3 shows the incident, refracted, transmitted, internally reflected and back-scattered rays and wavefronts. The lensing effect is so strong that the main focus of the lens begins inside the volume at the caustic of the refracted wave. It continues on the outside as the caustic of the transmitted rays and also on the inside as the first caustic of the internally reflected rays, in regions so small that they are barely visible at the left boundary of the volume. The end of the focus is a pair of arêtes on the axis of symmetry just inside and outside of the downstream boundary, respectively. A second caustic of the internal reflected waves, which also extends to the outside as the caustic of the back-scattered rays, is seen on the lower right-hand side of the volume. Consequently, the internal reflected wave first displays an inflection point and, later, a fold, the leading segment being remarkably straight. This wave is analogous to the rainbow wave in optics (Van de Hulst 1957). Its presence allows some liquid-filled spheres to be efficient sonar targets (Folds 1971).

More waves specific to a volume of low-sound-speed gas are shown on figure 4, where, in addition to the incident, refracted and transmitted waves, we include in the upper left corner of the volume the internal diffracted wave which is critically 


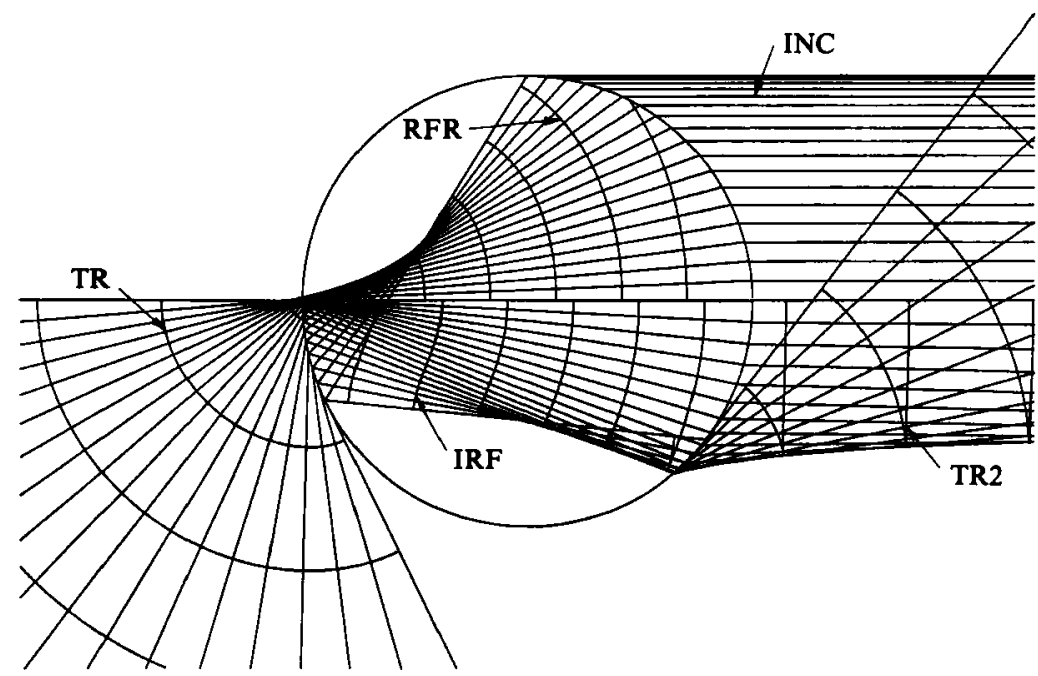

Flavre 3. Rays and wavefronts characteristic of the convergent case : INC, incident; RFR, refracted; TR, transmitted; IRF, internally reflected and TR2, back-scattered.

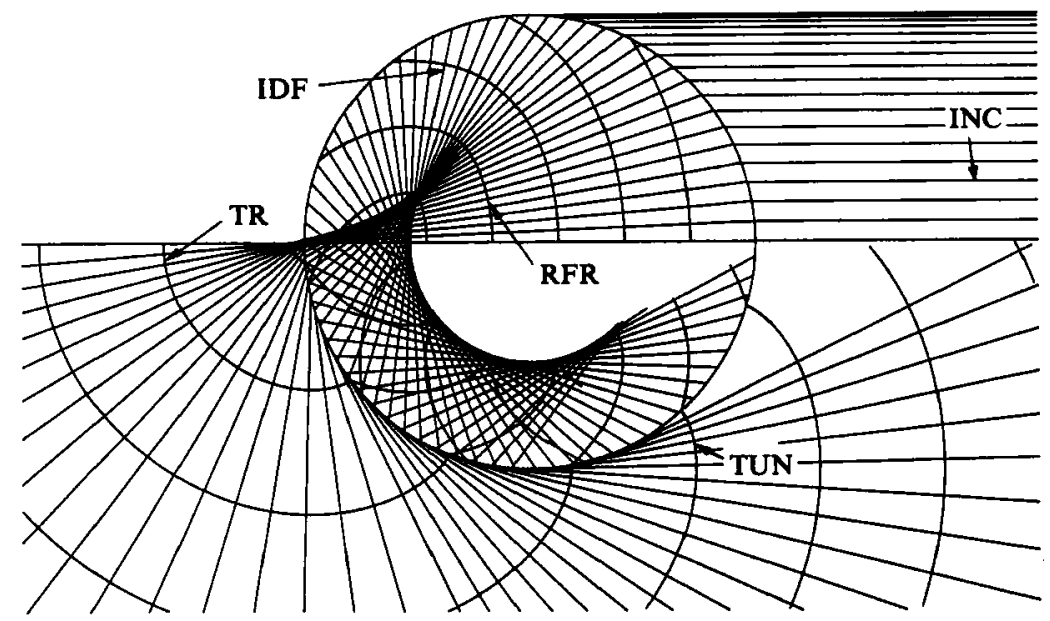

FraURe 4. Rays and wavefronts characteristic of the convergent case (contd): INC, incident; RFR, refracted; TR, transmitted; IDF, internally diffracted and TUN, tunnelled.

refracted into the cylinder from the external diffracted wave depicted in figure 1 . The rays of this wave form a circular caustic which originates at the arête of the caustic of the refracted wave. It is interesting that in this case the familiar crossing and folding that occurs downstream of the focus of an acoustic wave is confined within the acoustic lens, circling around the periphery of the lens indefinitely, while the main transmitted wave is without fold and nearly cylindrical. When the rays of the internal wave impinge on the boundary from the inside (at the critical angle) the transmission emerges from the volume as an evanescent wave. According to Jones (1978) it 'tunnels', and after a short distance propagates as a real wave again. We have been unable to positively identify these waves in our experiments (cf. §4).

The internal fronts, originally smooth, form two folds on two caustics. As the 


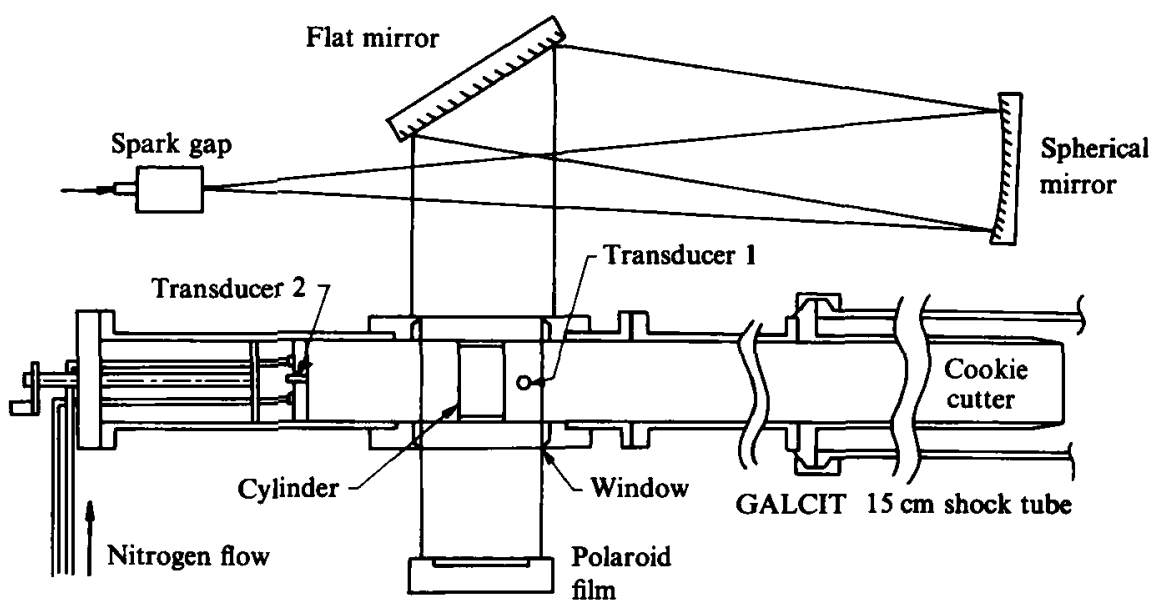

Figure 5. Plan view of the experimental set-up. Shock travels from right to left.

circular caustic never ends, the internal diffracted wave remains singly folded. Its leading (converging) segment is connected at the boundary to the external diffracted wave shown in figure 1 . Its trailing (focused) fold is the source of, and is connected at the boundary to, the evanescent wave. The evanescent wave joins at its other (outside) end with the transmitted wave, which, in contrast to the helium case, follows far behind the external diffracted wave. Otherwise, the evanescent wavefronts are identical in shape to the external diffracted waves.

In this case we distinguish between two families of backscattered waves. First, the waves resulting from the back reflection of the direct focused wave are very strong and they dominate. Second, the waves that are consequences of the external diffracted wave, though generated first, are much weaker. They are joined at their trailing edges to the strong family.

\section{Experimental set-up}

In the experiments shadowgraphy is used to exhibit the geometry of the wavefronts and the deformation of the volumes, and to obtain estimates of velocities, while pressure transducers are used to measure the strength of the waves and to determine the nature of the pressure field between the wavefronts.

\subsection{Shock tube}

The experiments are carried out in the GALCIT $15 \mathrm{~cm}$ diameter shock tube (Smith et al. 1967). A block diagram of the $8.9 \mathrm{~cm}$ square $\times 120 \mathrm{~cm}$ long test-section at the end of the shock tube is shown in figure 5. The test-section is matched to the circular cross-section of the basic facility with a $1.8 \mathrm{~m}$ long 'cookie-cutter' extension mounted inside the tube. In order to minimize the disturbance to the incident shock wave by the cookie cutter, the test-section walls were made thin, so the strength of the shock waves studied in the present work was limited to $M_{\mathrm{s}}<1.3$.

The motion of wavefronts and interfaces is recorded with a spark shadowgraph optical system through $15 \mathrm{~cm}$ diameter windows. The field of view in the photographs presented in this paper is approximately $95 \mathrm{~mm} \times 73 \mathrm{~mm}$. Only one photograph is taken during each run; a complete record of the wave pattern and of the deformation 
of an interface with increasing time is built up by delaying the photographs from an initial trigger signal, relying on the repeatability of conditions in the shock tube from run to run. With this method, clear photographs with good spatial resolution are obtained. However accuracy of the velocity measurements suffers: it is normally $10 \%$ or less but in some cases indicated in tables 2 and $4(\$ 6)$ it is degraded for a variety of reasons.

The initial trigger is obtained from a pressure transducer (transducer 1) mounted on the top wall of the test-section $64 \mathrm{~mm}$ upstream of the window centre. A piezoelectric transducer (transducer 2 ) is mounted in the centre of an instrument plate which acts as a movable endwall downstream of the viewing section. Thus transducer 2 measures the pressure as the waves downstream of the interaction reflect from the instrument plate. A survey of the wave processes is made by varying the distance between the instrument plate and the volume.

\subsection{Cylindrical volumes}

The test gas is contained within the cylindrical volume by a $0.5 \mu \mathrm{m}$ thick nitrocellulose membrane wrapped on $5 \mathrm{~cm}$ diameter $3 \mathrm{~mm}$ thick Pyrex windows which serve as the ends of the $8.8 \mathrm{~cm}$ long cylinder. The membranes are made from microfilm solution commercially available for indoor model airplanes. They are somewhat heavier $\left(50 \mu \mathrm{g} / \mathrm{cm}^{2}\right)$ than the range of films $\left(5-30 \mu \mathrm{g} / \mathrm{cm}^{2}\right)$ used in previous studies of the refraction of shock waves at plane interfaces (Jahn 1956; Abd-el-Fattah et al. 1976), in part because of the strain imposed during the assembly of the cylinders by the wrapping operation. Test gas is circulated continuously within the cylinder through its support structure. This flow creates a slight overpressure in the cavity which stretches the membrane into its cylindrical shape. Leakage and diffusion through the film are often sufficient that undesirable sound-speed gradients exist outside the cylinder unless even mixing in the test-section is promoted by small nitrogen jets issuing from the shock tube end plate. Leakage into the cylinder also perturbs the test-gas properties, but its influence does not vary substantially from run to run, so corrections can be made (cf. below). The cylindrical support structure can be located between the shock tube windows and at various distances upstream in order to observe the wave field and the behaviour of the gas volumes at later time, downstream of the initial position of the cylinder. The pressure history of the internal waves in the cylinder is measured using a modified cylindrical cell in which the pressure transducer is mounted in the centre of a wide and thick plate replacing the narrow connecting beam of the standard cylinder.

Spurious effects of the cylindrical cell on the shock wave propagation are due to the various support parts (end windows, connecting beam, etc.) and to the membrane. A measurement of the perturbations reflected upstream was made with the upstream transducer. For a shock wave of Mach number 1.09 incident on a nitrogen-filled cylinder, three short pressure pulses were observed on the pressure plateau behind the incident shock. The first one, corresponding to a wave of Mach number of 1.005, is due to the membrane, and the following two pulses, each from waves of Mach number about 1.01, are due to the waves reflected from the end windows and the connecting beam.

\subsection{Spherical volumes}

Spherically-shaped volumes of test gas are made by blowing $4.5 \mathrm{~cm}$ diameter soap bubbles from a small support structure centred in the test-section of the shock tube. In previous experiments (Rudinger 1958; Davy \& Blackstock 1971) the soap bubbles 


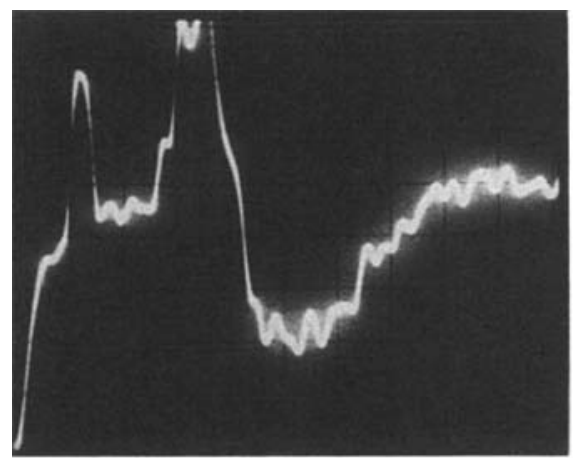

(a)

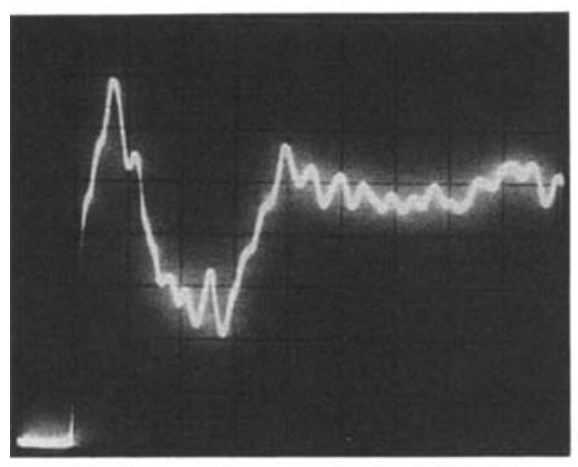

(c)

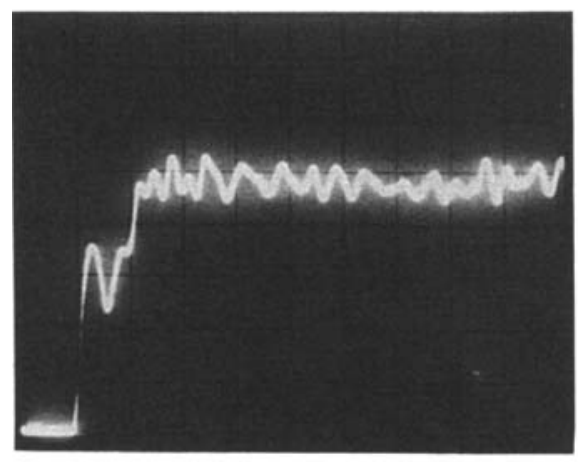

(e)

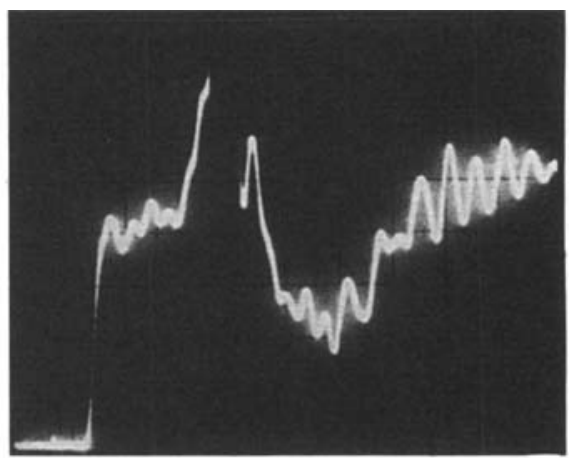

(b)

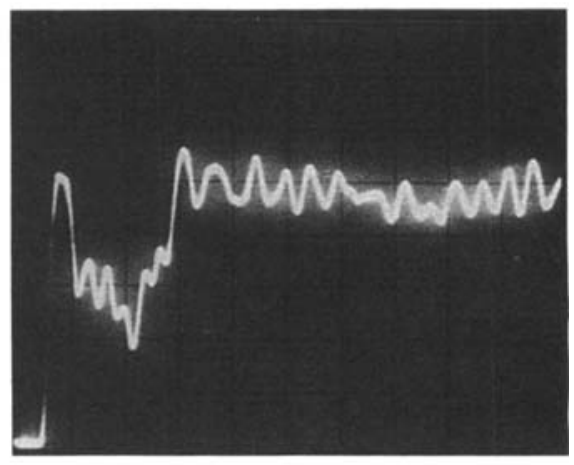

(d)

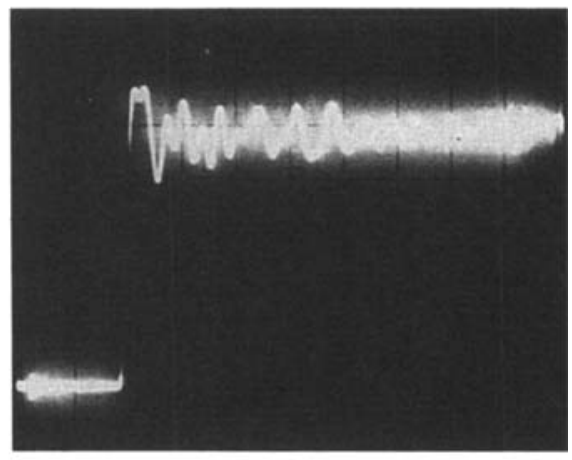

(f)

Figtre 6. Pressure field due to an $M_{\mathrm{s}}=1.25$ shock wave at various distances downstream of a $4.5 \mathrm{~mm}$ diameter spherical volume of $\mathrm{a} \frac{1}{3}$ helium $\frac{2}{3}$ argon mixture. (a) distance to sphere: $1 \mathrm{~mm},(b)$ $4 \mathrm{~mm},(c) 14 \mathrm{~mm},(d) 24 \mathrm{~mm},(e) 34 \mathrm{~mm},(f) 44 \mathrm{~mm}$. Vertical scale: $380 \mathrm{mbar} /$ div, horizontal scale: $5 \mu \mathrm{s} /$ div.

were held on a ring. In the present configuration the bubbles are suspended from a $6 \mathrm{~mm}$ diameter cup, where a drop of soap is initially deposited, at the end of a $0.75 \mathrm{~mm}$ diameter stainless steel tube through which the test gas is injected. The bubble film is made of Plateau's soap solution (78\% distilled water, $2 \%$ sodium oleate and $20 \%$ glycerine by mass). It varies in thickness, depending on conditions, from 
about $0.25 \mu \mathrm{m}$ to $1.0 \mu \mathrm{m}$. Helium-filled bubbles, floating up from the support tube, are nearly spherical and have the thinnest membranes. All other bubbles hang down from the support tube. They are also nearly spherical except for the R22-filled bubbles which are elongated (height $35 \mathrm{~mm}$, width $30 \mathrm{~mm}$ ) because of their high density. The initial position of the bubbles can also be set upstream of the shock-tube windows.

Due to reduced optical depth the image of the wave pattern in the axisymmetric configuration appears weaker on the shadowgraphs than in the cylindrical configuration. On the other hand, due to $(a)$ its less intrusive support structure, $(b)$ the smaller volume of gas relative to the size of the test-section, and (c) the fact that after shock passage the film ruptures in a more ideal fashion, the interaction is less affected by spurious effects. However, it should be noted that the relatively small effect of the structure is locally magnified near the axis in the axisymmetric case by focusing (forward glory). Figure 6 shows some pressure traces recorded just behind bubbles filled with a $\frac{1}{3}-\frac{2}{3}$ mixture of helium and argon which is acoustically equivalent to air but has a different optical index of refraction for flow visualization. The incident shock Mach number is 1.25 . The sequence of traces exhibits the various waves and how they merge. In the trace at $1 \mathrm{~mm}$, the initial rise is caused by the transmitted wave, while the first peak is due to the wave diffracted into the shadow region of the soap film. The large second peak is the signature of the secondary transmitted wave (forward glory) arising from the reflections of the internal wave from the film. The subsequent pressure variations after the second peak may be due to later (tertiary, etc.) transmitted waves. At $4 \mathrm{~mm}$, only the glory is seen behind the initial front. Its subsequent merging with the primary transmitted shock is shown in the following traces. By $44 \mathrm{~mm}$, the merging process is nearly complete and the pressure profile is similar to the profile of an undisturbed shock wave.

\section{Results: flow visualization}

\subsection{Divergent case}

In this section we present a sequence of photographs which illustrates the wave patterns induced by shock interaction with a divergent acoustic lens and the consequent deformation and mixing of the inhomogeneity.

\subsubsection{Cylinder}

Figure $7(a)$ is a view of the helium cylinder $32 \mu$ s after a shock wave of strength $M_{\mathrm{s}}=1.22$ incident from the right impacts the volume. The circular shape is the outline of the Pyrex windows at the ends of the cylinder. The T-shaped object at the bottom is the structure which supports the windows. The incident shock appears as two straight branches connected on the cylinder to the curved refracted wave (on the left) and reflected wave (on the right). At the time of the photograph transition between regular and irregular refraction is taking place, and the upstream (right) interface has already been set into motion.

Figure $7(b)$ at $52 \mu \mathrm{m}$, shows the refracted wave running out far ahead, connected at the interface to the two branches of the transmitted wave which cross the two branches of the incident wave and join tangentially the reflected wave. The transmitted wave forms a precursor to the incident wave. The upstream air-helium interface has flattened. At $62 \mu \mathrm{s}$ (figure 7c) the transmitted wave emerges entirely from the left-hand interface, and the converging internal reflected wave appears faintly with two cusps. At the top of the picture in air is an interesting example of 

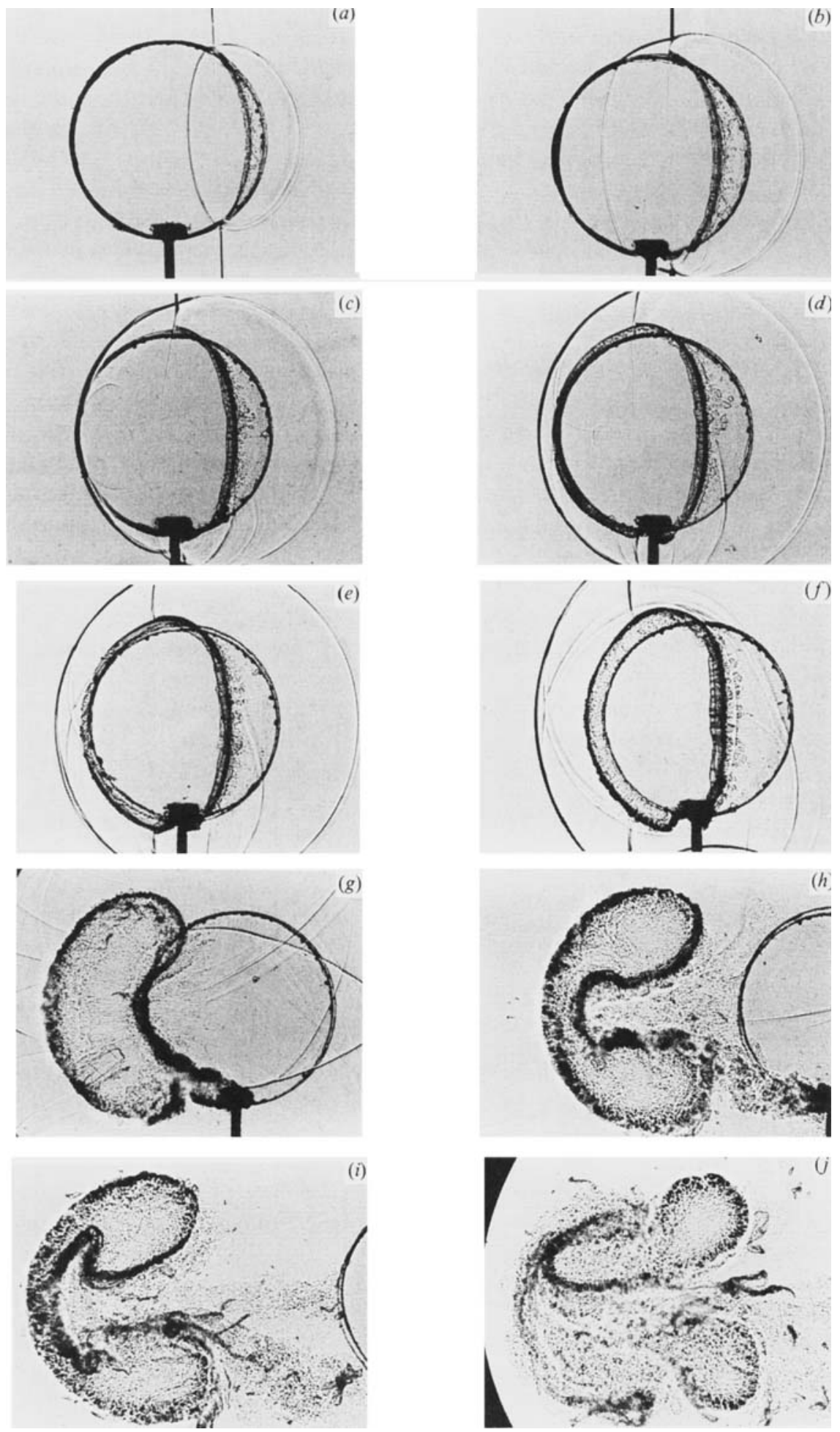

Figure 7. Shadow-photographs of the interaction of an $M_{\mathrm{s}}=1.22$ shock wave moving from right to left over a cylindrical helium volume (5 cm diameter). (a) $t=32 \mu \mathrm{s},(b) 52 \mu \mathrm{s},(c) 62 \mu \mathrm{s},(d) 72 \mu \mathrm{s}$, (e) $82 \mu \mathrm{s},(f) 102 \mu \mathrm{s},(g) 245 \mu \mathrm{s},(h) 427 \mu \mathrm{s},(i) 674 \mu \mathrm{s},(j) 983 \mu \mathrm{s}$. 

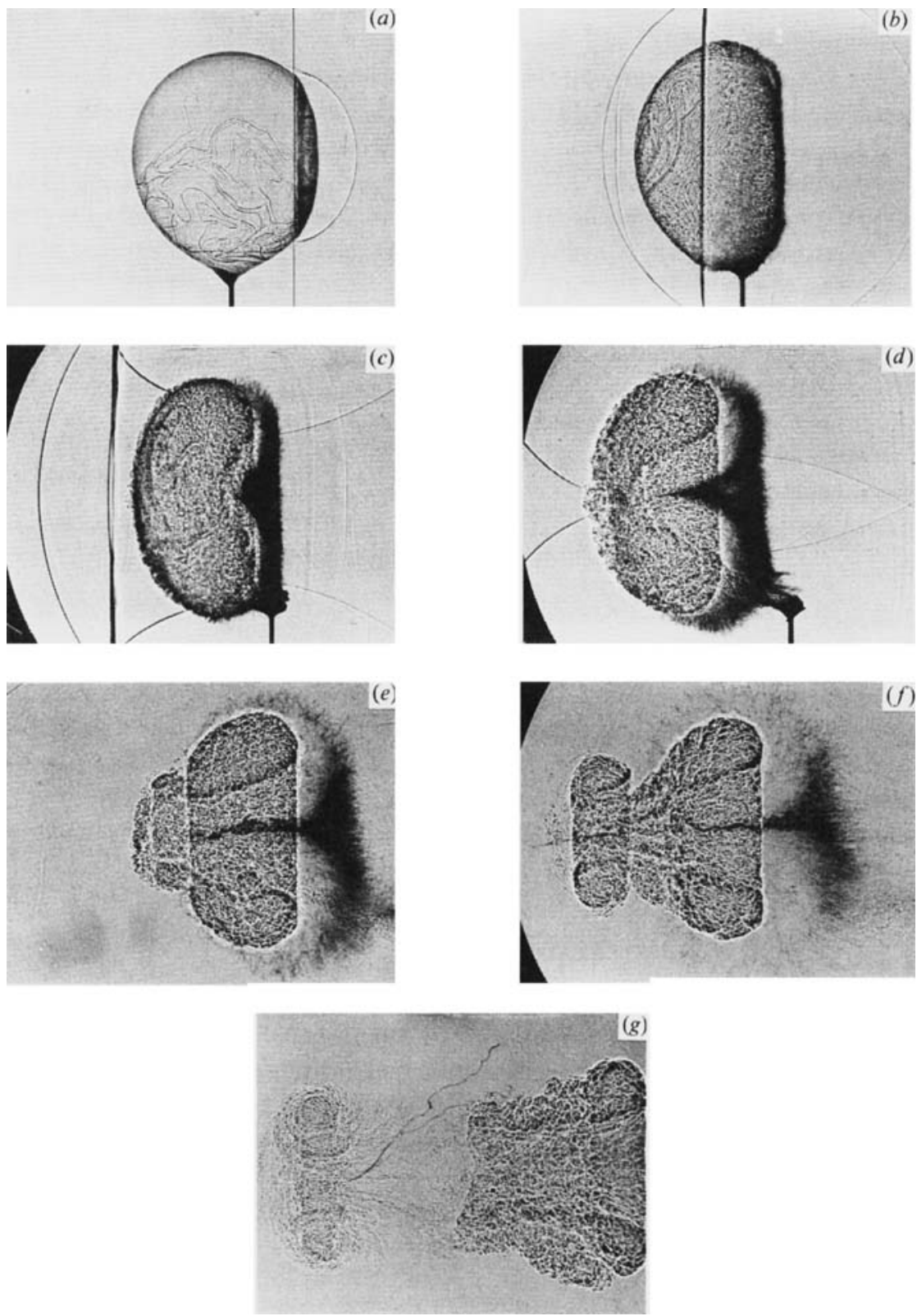

Figure 8. Shadow-photographs of the interaction of an $M_{\mathrm{s}}=1.25$ shock wave moving from right to left over a spherical helium volume (4.5 cm diameter). (a) $t=20 \mu \mathrm{s},(b) 82 \mu \mathrm{s},(c) 145 \mu \mathrm{s},(d)$ $223 \mu \mathrm{s},(e) 350 \mu \mathrm{s},(f) 600 \mu \mathrm{s},(g) 1594 \mu \mathrm{s}$.

a shock-on-shock interaction, which forms a quadruple shock intersection. At $72 \mu s$ (figure $7 d$ ), the secondary transmitted waves are seen outside the volume tangentially connected to the (primary) transmitted wave and connected at the interface to the internal reflected wave, now approaching its focus. A wave reflected from the transversal beam is seen at bottom right. At $82 \mu \mathrm{s}$ (figure $7 e$ ), the internal reflected wave has become a diverging wave, having passed through its caustic. Outside, the 
two branches of the secondary transmitted wave intersect each other on the centreline.

At $102 \mu$ s (figure $7 f$ ), small portions of the incident shock are visible at the top and bottom of the photograph, diffracting into the shadow region downstream of the cylinder. The primary and secondary transmitted waves are almost entirely merged, while the internal reflected wave has just emerged from the upstream interface as the back-scattered wave. The wave reflected from the connecting beam is still visible at the far right, while the wave appearing at the bottom has been reflected from the shock-tube wall. Note that, though the incident shock is still passing over the body, considerable distortion and motion of the helium volume has already occurred: the upstream interface is almost flat, and the body has expanded laterally as a consequence of the shock interaction.

As time passes, the upstream face continues to deform, and after $245 \mu$ s the volume has acquired a kidney shape, as shown in figure $7(g)$. Figure $7(h-j)(427 \mu \mathrm{s}, 674 \mu \mathrm{s}$ and $983 \mu \mathrm{s}$, respectively) shows how a re-entrant jet forms. When the head of the jet impinges on the downstream high-density air interface it spreads out laterally, eventually forming a pair of ill-defined vortical structures.

\subsubsection{Sphere}

Figure $8(a)$ shows an $M_{\mathrm{s}}=1.25$ incident shock wave $20 \mu$ s after its collision with a buoyant helium-filled soap bubble floating above its support tube. Barely visible inside the bubble in the original photograph is the refracted wave, which has run well ahead of the incident shock. The soap film at the upstream face is seen to be disintegrating. The dark striations on the surface of the bubble are due to variations of the film thickness induced by surface-tension gradients due to evaporative cooling before shock arrival. Figure $8(b)(82 \mu \mathrm{s})$ shows the spherical transmitted wave at the left immediately followed by a flat front which is the projection of the torus-shaped secondary transmitted wave (forward glory).

Figure $8(c)(145 \mu \mathrm{s})$ shows, left to right, the transmitted wave which has been caught up by the glory wave and the diffracted wave just after emerging from the helium volume. The back-scattered wave to the right of the volume is the internal reflected wave which has crossed the flat upstream interface to the exterior. The other wave seen faintly just $t, o$ its left is a back-scattered wave resulting from the second internal reflection, i.e. backward glory. Reflections of the reflected wave from the shock tube walls appear at the top and bottom. A fine-scale Rayleigh-Taylor instability is seen developing on the surface of the sphere, especially at the downstream interface. The black band at the right-hand side of the picture is a finely dispersed aerosol of the soap film which, by its inertia, lags behind the main volume. The aerosol forms a useful marker of the air from the upstream side of the flow, which is entrained into the volume by the re-entrant jet of air (figure $8 d, 223 \mu \mathrm{s}$ ). The air jet impinges on the downstream helium-air interface and pierces it (figure $8 e, 350 \mu \mathrm{s}$ ), forming a more distinct vortex ring than in the cylindrical case (figure $8 f, 600 \mu \mathrm{s}$ ). The small scales evident on the vortex ring at later times (figure $8 g, 1594 \mu \mathrm{s}$ ) indicate that it contains most of the vorticity in the flow, and is the region in which the most intense mixing takes place. Concentrated vorticity also occurs along the conical shear layer at the boundary of the re-entrant jet in the main body (cf. below). Figures $8(f)$ and $8(g)$ show slender filaments of soap that have been projected along the axis all the way to the front of the structure and then around the core of the vortex ring. Both the cylindrical and spherical sequences offer some analogies with the buoyancy- 


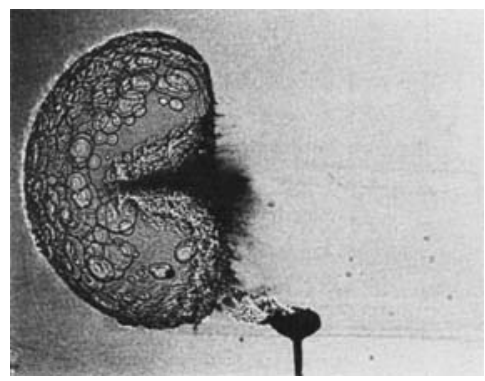

Figure 9. Shadow-photograph of an initially spherical helium volume $(4.5 \mathrm{~cm}$ diameter $), 0.51 \mathrm{~ms}$ after interaction with an $M_{\mathrm{s}}=1.10$ shock wave moving from right to left.

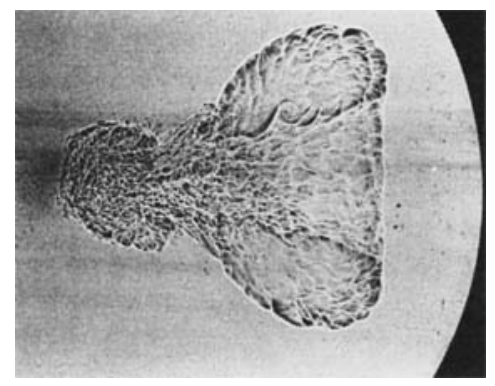

FIGURE 10. Shadow-photograph of an initially spherical helium volume $(4.5 \mathrm{~cm}$ diameter $), 2.64 \mathrm{~ms}$ after interaction with an $M_{\mathrm{s}}=1.05$ shock wave moving from right to left.

driven deformation of cylindrical and spherical air bubbles in water (cf. Walters \& Davidson 1962, 1963).

Figure 9, taken $0.51 \mathrm{~ms}$ after interaction with a weaker $\left(M_{\mathrm{s}}=1.10\right)$ shock wave, more clearly shows the spike of air driven through the helium volume, entraining the remnants of the upstream soap membrane. Fine-scale turbulence is seen on the conical shear layer. The circles appearing on the left-hand side of the volume in figure 9 are holes in the soap membrane which had been created about $0.2 \mathrm{~ms}$ earlier.

An even weaker shock wave $\left(M_{\mathrm{s}}=1.05\right)$ creates a yet slower air jet within the helium volume, with a smaller vortex ring and less small-scale turbulence. Characteristic large-scale vortical structures seen in figure $10(2.64 \mathrm{~ms})$ identify the conical shapes as classical free shear layers on the re-entrant jet. The visual thickness of these shear layers $(\delta / x \approx 0.2$ to 0.33$)$ is consistent with the results of Brown \& Roshko (1974 figure 7) for the estimated conditions of the present experiments, namely, density ratio 5-7 and velocity ratio $2-3$ between the high-speed air and the low-speed helium flows.

\subsubsection{Cylinder}

\subsection{Convergent case}

A cylindrical volume of $\mathrm{R} 22$ is such a strong convergent acoustic lens that shockwave focusing is the dominant feature observed in the wave patterns. Figure $11(a)$ $(55 \mu \mathrm{s})$ shows the initial stages of the interaction of an $M_{\mathrm{s}}=1.22$ shock wave with a low-sound-speed inhomogeneity. The incident and the reflected shock waves are seen outside the cylinder and the convergent refracted shock inside. The two extremities 

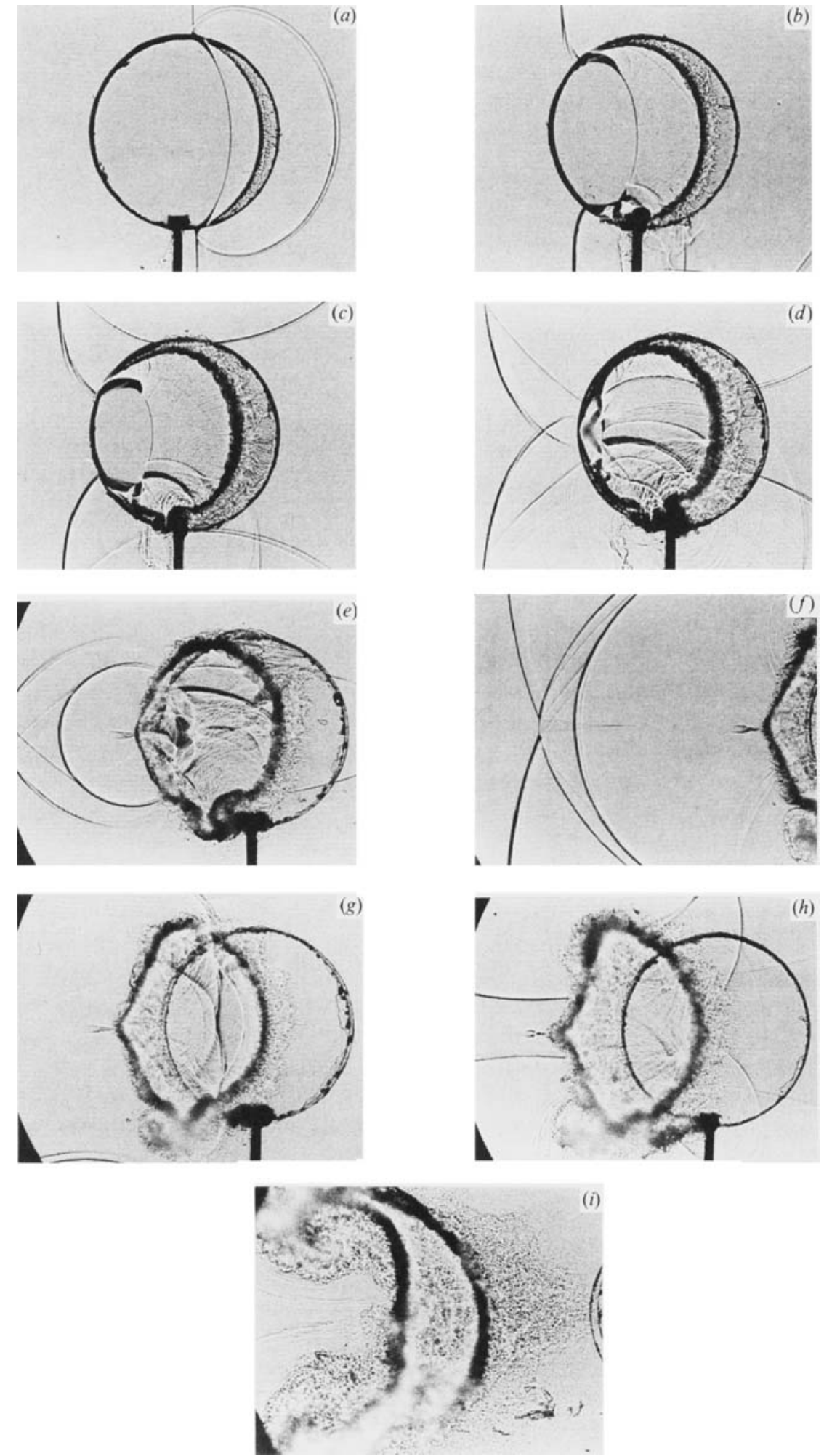

FIGURE 11. Shadow-photographs of the interaction of an $M_{\mathrm{s}}=1.22$ shock wave moving from right to left over a cylindrical R22 volume (5 cm diameter). (a) $t=55 \mu \mathrm{s},(b) 115 \mu \mathrm{s},(c) 135 \mu \mathrm{s},(d) 187 \mu \mathrm{s}$, (e) $247 \mu \mathrm{s},(f) 318 \mu \mathrm{s},(g) 342 \mu \mathrm{s},(h) 417 \mu \mathrm{s},(i) 1020 \mu \mathrm{s}$. 
of the refracted front, generated here by a grazing incident shock, appear very thick in the image. The upstream interface has moved away from its initial position on the cylindrical frame. In figure $11(b)(115 \mu \mathrm{s})$ the incident shock has diffracted into the shadow of the cylinder and is connected to the refracted wave inside by two black bands which are the internal diffracted wave fronts. The contrast between the thin refracted wave and the thick internal diffracted wave is striking. At $135 \mu$ s (figure $11 \mathrm{c})$ the contrast between the refracted and diffracted fronts inside is amplified. This is about the time that the formation of two caustics at an arête at the junction between the two waves is expected. Visible at the top and bottom of the figure are the reflections of the reflected wave from the test-section walls. The waves reflected from and diffracted around the transversal beam can also be seen in the lower part of the photograph. Note that the volume of the inhomogeneity has been substantially reduced by shock compression.

At $187 \mu$ s (figure $11 d$ ), the two branches of each set of diffracted waves have crossed, and the refracted wave front is approaching its focus near the downstream air-R22 interface. It then expands radially outside the cylinder, as shown in figure $11(e)(247 \mu \mathrm{s})$. The crossed waves which it has overtaken outside are spurious, the reflected waves from the bottom and top of the test-section. Still ahead are the external diffracted waves. A slender hot focal region is seen just to the left of the downstream interface. High velocity created by the transmitted shock at its focus causes a central wedge to form on the downstream R22-air interface (figures $11 e$ and 11f). At $318 \mu$ s (figure $11 f$ ) the expanding transmitted wave has almost caught up with the diffracted wave. The crossing of the two oblique branches of the diffracted wave has led to the formation of a vertical Mach shock and two slipstreams forming a triangle on the axis. This configuration was observed by Bryson \& Gross (1961), who named the features Mach shock 2 and contact discontinuity 2, respectively. At $342 \mu$ s (figure $11 \mathrm{~g}$ ) the internal back-reflected wave is visible while at $417 \mu \mathrm{s}$ (figure $11 \mathrm{~h}$ ) its exterior transmission as the backscattered wave can be seen. At later times, after the waves have passed from the picture, the structure deforms into a large vortex pair (figure $11 i, 1020 \mu \mathrm{s})$. Note how the body of fluid has grown laterally during the formation of the vortices. The two vortices grow in size by entraining the fluid left at the centre.

The same wave pattern and deformation history is observed in the interaction with a weaker wave $\left(M_{\mathrm{s}}=1.085\right)$. For a wave of this strength the rate of deformation of the volume is about half as fast as for the $M_{\mathrm{s}}=1.22$ case. Figure $12(a)$, taken $156 \mu \mathrm{s}$ after the initial contact, highlights again the difference between the internal refracted and diffracted waves. The nature of the two successive reflected wave fronts propagating to the right inside the cylinder in figure $12(b)(279 \mu \mathrm{s})$ is not understood, but we speculate that it is due to the shock-wave thickening and folding process that occurs after propagation through a caustic (Sturtevant \& Kulkarny 1976). For this shock strength the two slipstreams defining the hot spot at the focus merge on both sides of the spot, while in the case of the stronger shock (figures $11 e$ and $11 f$ ) they were open on the left-hand side. A similar behaviour was observed by Sturtevant \& Kulkarny (1976) in the case of shock-wave focusing by cylindrical reflectors.

\subsubsection{Sphere}

Though the response to shock excitation of soap bubbles filled with $\mathrm{R} 22$ is basically the same as cylinders, many of the details seem to be qualitatively different. Because of the high density of R22, the 'spheres' are in fact oval bubbles hanging down from the support tube. In figure 13 the refracted wave inside the R22 bubble can be seen 

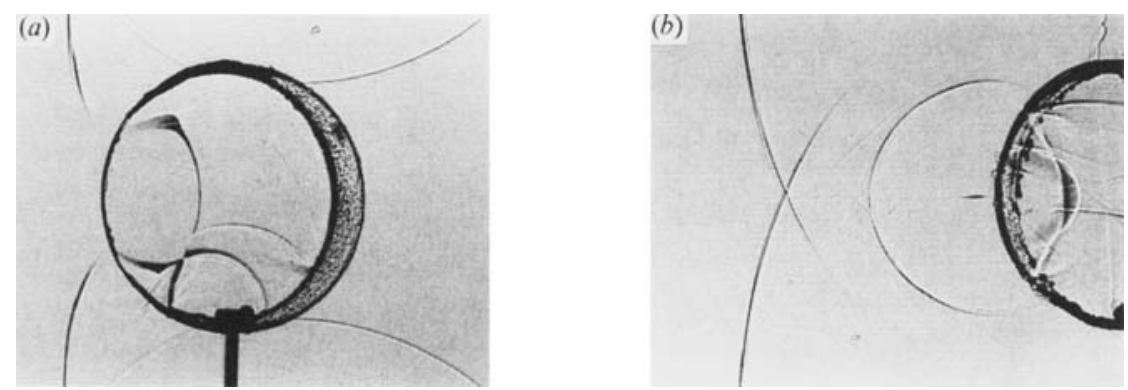

FIGURE 12. Shadow-photographs of the interaction of an $M_{\mathrm{s}}=1.085$ shock wave moving from right to left over a cylindrical R22 volume (5 cm diameter). (a) $t=156 \mu \mathrm{s},(b) 279 \mu \mathrm{s}$.

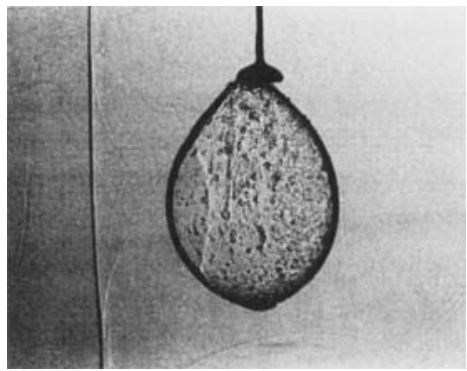

Figure 13. Shadow-photograph of the interaction of an $M_{\mathrm{s}}=1.07$ shock wave moving from right to left over a R22 sphere ( $3.5 \mathrm{~cm}$ high, $2.5 \mathrm{~cm}$ wide), $t=138 \mu \mathrm{s}$.
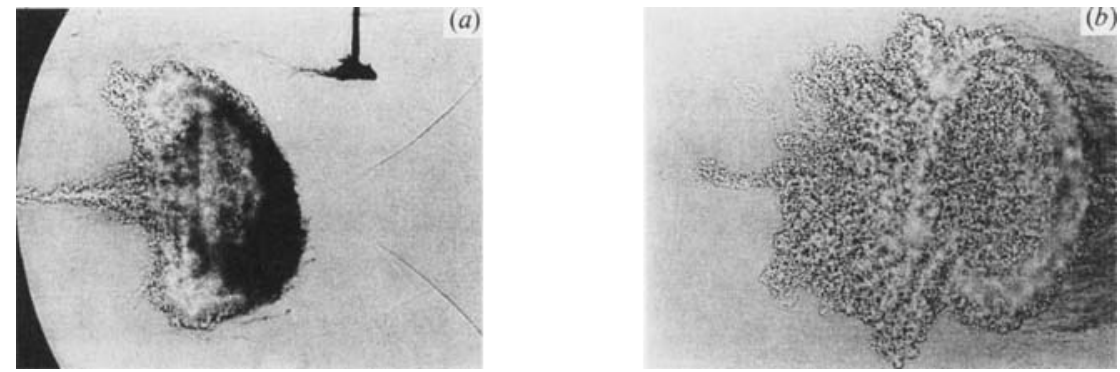

Figure 14. Shadow-photographs of a R22 sphere (3.5 cm high, $2.5 \mathrm{~cm}$ wide) after interaction with an $M_{\mathrm{s}}=1.25$ shock wave moving from right to left; $(a) t=507 \mu \mathrm{s},(b) 1.56 \mathrm{~ms}$.

$138 \mu \mathrm{s}$ after impact of a rather weak shock $\left(M_{\mathrm{s}}=1.07\right)$. From figure $14 a\left(M_{\mathrm{s}}=1.25\right.$, $507 \mu \mathrm{s})$ it is seen that deformation of the wedge created by the transmitted wave near its focus, ultimately forming a narrow axial jet, is much more extreme than in the cylindrical case, presumably because of the stronger focus in axial symmetry. On the other hand, the surrounding vortex ring, which in cylindrical symmetry is a vortex pair, is much more diffuse. Indeed, at late times (figure $14 b, 1.56 \mathrm{~ms}$ ), the structure at the left expands, becoming sinuous and even more diffuse. 


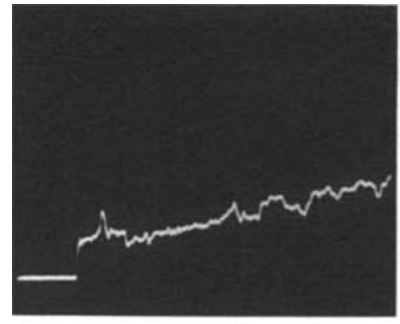

(a)

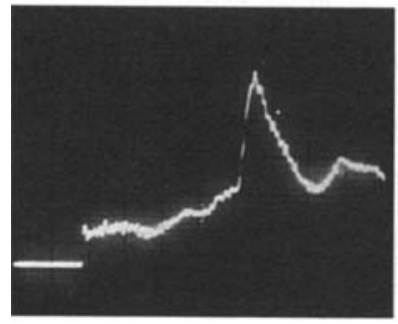

(d)

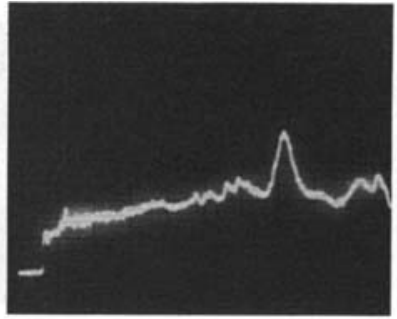

(b)

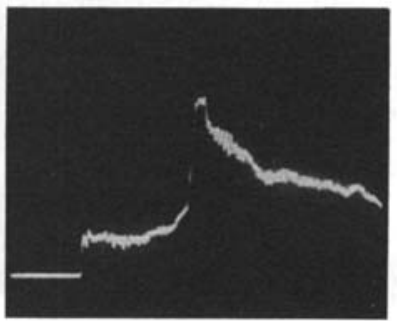

(e)

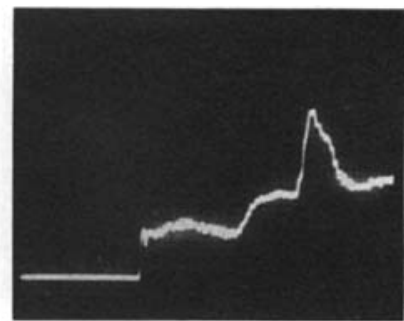

(c)

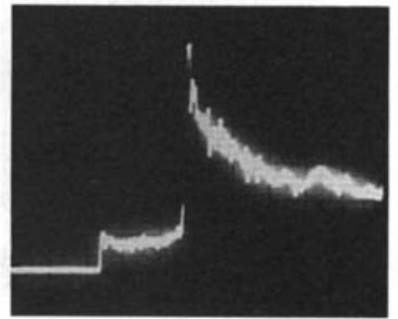

(f)

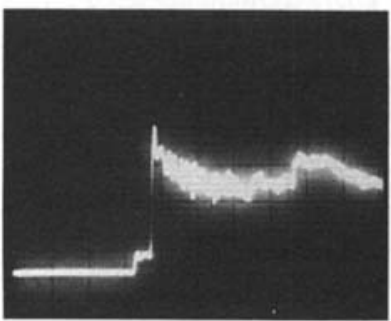

$(g)$

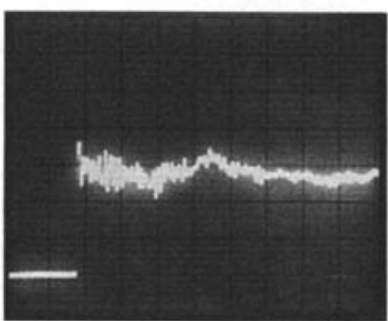

(h)

Fiqure 15. Pressure profiles recorded downstream of the helium eylinder for an $M_{\mathrm{s}}=1.085$ incident shock at several locations behind the cylinder. Distances to the cylinder are: $(a) 3 \mathrm{~mm},(b) 19 \mathrm{~mm}$, (c) $38 \mathrm{~mm},(d) 59 \mathrm{~mm},(e) 81 \mathrm{~mm},(f) 121 \mathrm{~mm},(g) 229 \mathrm{~mm}$ and $(h) 497 \mathrm{~mm}$. Horizontal scale: $20 \mu \mathrm{s} / \mathrm{div}$, vertical scale: $190 \mathrm{mbar} / \mathrm{div}$.

\section{Results: pressures}

\subsection{Divergent case}

Pressure profiles for a $M_{\mathrm{s}}=1.085$ shock wave incident on a helium-filled cylinder measured at various distances behind the cylinder with a transducer mounted in the centre of the instrument plate are shown in figure 15. The behaviour of an $M_{\mathrm{s}}=1.22$ shock is qualitatively the same. The signal begins with the transmitted wave, which causes a rapid jump of pressure. In the field between the transmitted wave and the diffracted wave there is a gradual increase of pressure. The triangular peak which follows is the signature of the diffracted wave. Initially it has a relatively slow rise and symmetric fall. The diffracted wave does not appear on the first trace $(3 \mathrm{~mm})$; in that figure the small blip which occurs about $15 \mu$ s after the transmitted shock is, in fact, the secondary transmitted wave. As shown at the downstream stations, 


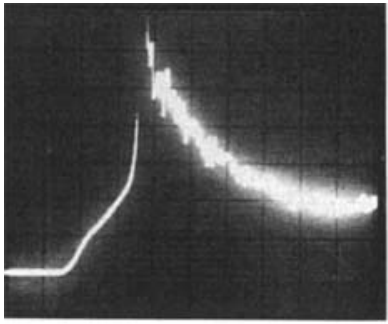

(a)

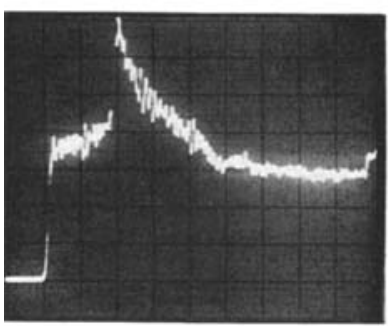

(d)

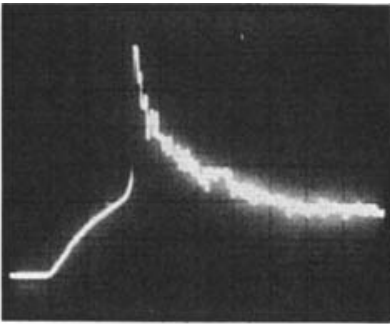

(b)

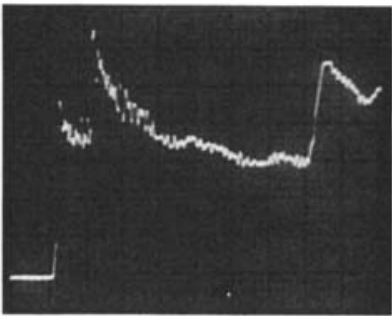

(e)

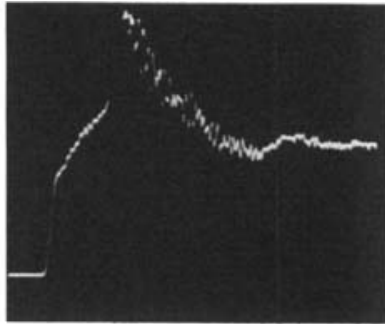

(c)

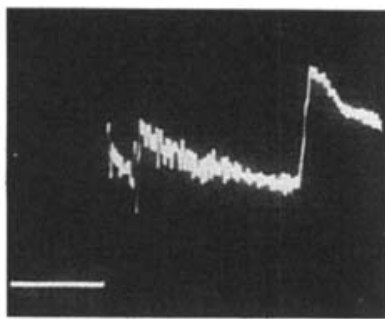

(f)

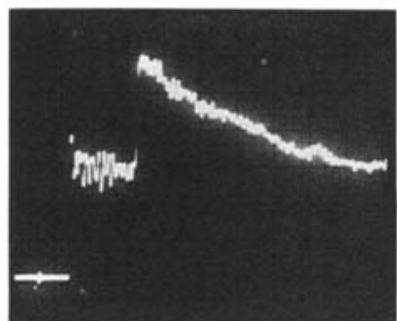

(g)

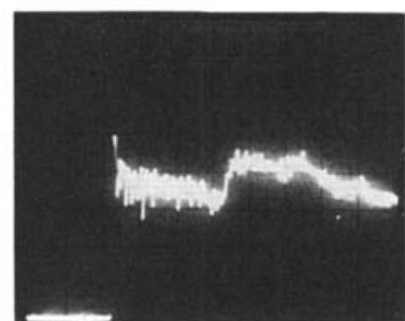

(h)

Figure 16. Pressure profiles recorded downstream of the R22 cylinder for an $M_{\mathrm{s}}=1.22$ incident shock at several locations behind the cylinder. Distances to the cylinder are: $(a) 3 \mathbf{m m},(b) 11 \mathrm{~mm}$, (c) $27 \mathrm{~mm},(d) 43 \mathrm{~mm},(e) 67 \mathrm{~mm},(f) 99 \mathrm{~mm},(g) 180 \mathrm{~mm}$ and $(h) 329 \mathrm{~mm}$. Horizontal scale: $20 \mu \mathrm{s} / \mathrm{div}$, vertical scale: $(a, b) 760 \mathrm{mbar} / \mathrm{div},(c)$ to $(h) 380 \mathrm{mbar} / \mathrm{div}$.

\begin{tabular}{lllllllll}
\hline Gas & $M_{\mathrm{s}}$ & $M_{\mathrm{D}}$ & $M_{\mathrm{R}}$ & $M_{\mathrm{R} 1}$ & $M_{\mathrm{T}_{1}}$ & $M_{\mathrm{Tf}}$ & $M_{\mathrm{T} 1}$ & $x / R$ \\
$\mathrm{R} 22$ & 1.03 & 1.02 & - & 1.040 & 1.01 & - & 1.029 & - \\
& 1.085 & 1.07 & 1.17 & 1.114 & 1.065 & 1.01 & 1.080 & 12 \\
& 1.22 & 1.22 & 1.37 & 1.297 & 1.22 & 1.04 & 1.207 & 5 \\
Helium & 1.085 & 1.11 & 1.045 & 1.045 & 1.045 & 1.02 & 1.069 & 10 \\
& 1.22 & 1.15 & 1.08 & 1.114 & 1.12 & 1.10 & 1.177 & 6
\end{tabular}

$M_{\mathrm{s}}$, incident wave Mach number. $M_{\mathrm{D}}$, measured diffracted wave strength. $M_{\mathrm{R} 1}$, refracted wave strength calculated from one-dimensional theory. $M_{\mathrm{R}}$, refracted shock strength measured inside modified cylinder. $M_{\mathrm{T} 1}$, transmitted wave strength calculated from one-dimensional theory. $M_{\mathrm{T}_{\mathrm{i}}}$ measured transmitted wave strength close to cylinder. $M_{\mathrm{Tf}}$, measured transmitted wave strength just before merging. $x / R$, merging distance ( $R=$ cylinder radius).

TABLE 1. Experimental and theoretical shock strengths 


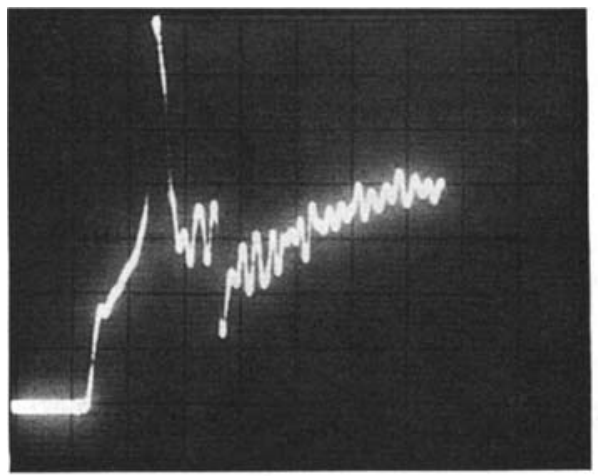

(a)

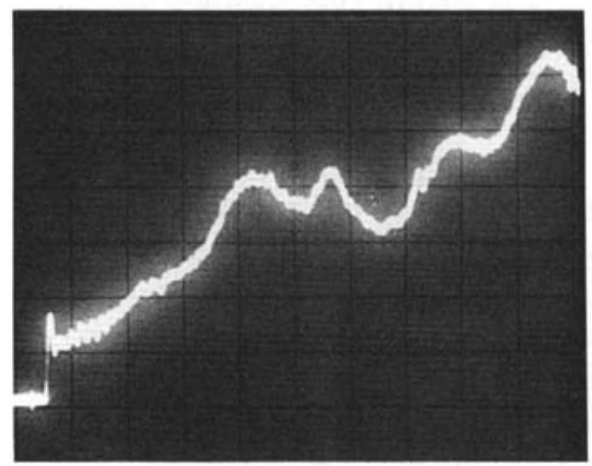

(c)

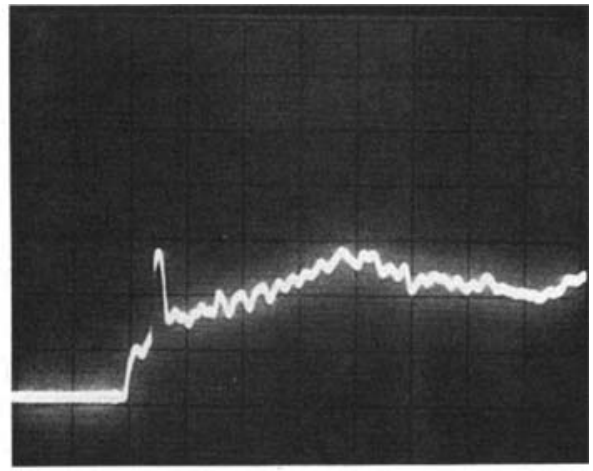

(b)

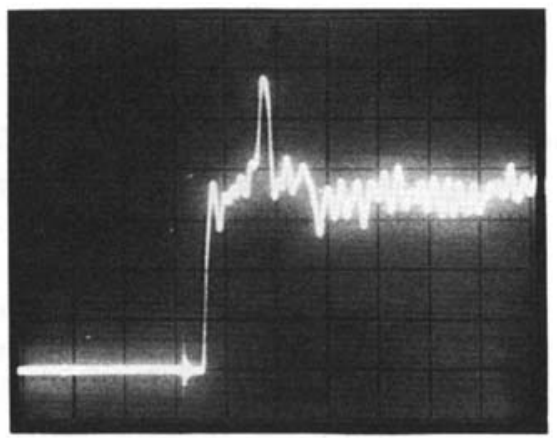

(d)

Figure 17. Pressure profiles recorded very close to the helium spheres: $(a, b, c)\left(M_{\mathrm{s}}=1.25\right)$ and helium cylinder $(d)\left(M_{\mathrm{s}}=1.22\right)$. Distances are: $(a) 2 \mathrm{~mm},(b) 11 \mathrm{~mm},(c) 25 \mathrm{~mm}$ and $(d) 3 \mathrm{~mm}$. Horizontal scale: $(a, b, d) 10 \mu \mathrm{s},(c) 20 \mu \mathrm{s}$, vertical scale : $(a, d) 190 \mathrm{mbar} / \mathrm{div},(b, c) 280 \mathrm{mbar} / \mathrm{div}$.

the diffracted wave steepens to become a shock front followed by a rapid expansion within a distance of $81 \mathrm{~mm}$, and this shock, in turn, catches up with the transmitted wave about $250 \mathrm{~mm}$ downstream of the cylinder.

\subsection{Convergent case}

The pressure measurements for the case of a relatively strong shock incident on the R22-filled cylinder (figure 16) show that close to the cylinder the first disturbance (the diffracted wave) is smooth. It is followed by the (discontinuous) transmitted wave which is very strong, carrying overpressures up to 6.7 bar near its focus. The diffracted wave steepens $(27 \mathrm{~mm})$, becoming a shock $(43$ and $67 \mathrm{~mm})$ and is caught by the transmitted shock $99 \mathrm{~mm}$ behind the cylinder. Henceforth the initial disturbance is the combined front. The pulses seen behind the combined front on the pressure traces from $99 \mathrm{~mm}$ on are due to various waves reflected from the shock-tube sidewalls, the strongest one being the reflection of the transmitted wave from the top and bottom walls. It is worth noting the similarity of the pressure profiles obtained near the cylinder to the ones reported by Sturtevant \& Kulkarny (1976 figure 7) in their study of shock focusing. 


\subsection{Wave strengths: cylinders}

Measured pressure jumps from profiles similar to those shown above have been used to calculate the strength (i.e. local Mach number) of the various waves in the cylindrical configuration. In table 1 the results are compared with the wave strengths resulting from the (one-dimensional) interaction of a shock wave with a layer of helium or R22 between plane interfaces parallel to the shock wave calculated by one-dimensional gasdynamics. Downstream of merging the Mach number of the combined diffracted and refracted wave was found to be very close to that of the incident shock Mach number. In R22 the transmitted wave strength decreases significantly between the focal area and the merging point. The calculated values in the table are for the interaction of a plane shock wave with a parallel plane gaseous interface, and are not expected to compare well when effects of wave curvature are large. The converging refracted wave in $\mathrm{R} 22$ is expected to be stronger and the diverging refracted wave in helium should be weaker than the one-dimensional value. (Conversely, contamination by air of the test gas within the cylinder will lead to a stronger refracted wave in helium and a weaker one in $\mathrm{R22}$, cf $\S 6$.)

\subsection{Forward glory from the helium spheres}

The three pressure profiles of figures $17(a-c)$ were obtained for a strong incident shock wave $\left(M_{\mathrm{s}}=1.25\right)$ with the transducer located very close behind $(2,11$ and $25 \mathrm{~mm})$ the downstream edge of a helium-filled bubble. The first pressure profile indicates a transmitted shock of strength $M_{\mathrm{s}}=\mathbf{1 . 0 6}$ followed by a strong $\mathbf{N}$-shaped pulse of about 1.24 bar amplitude, which is the secondary transmitted wave (forward glory). The second and third profiles show how the secondary transmitted wave merges with the (primary) transmitted wave. The third profile also shows the pressure rise due to the diffracted wave. As shown by the fourth profile $(d)$, recorded $3 \mathrm{~mm}$ behind the helium cylinder with an $M_{\mathrm{s}}=1.22$ incident shock, the forward glory is much weaker in the cylindrical configuration than in the spherical.

\section{Results: velocities}

The velocities of the shock waves and gas interfaces observed in the shadowgraphs have been estimated by plotting their location against time ( $x$ vs. $t)$ and calculating the slopes of straight lines fitted to the data. The positions of the refracted waves, transmitted waves, and interfaces are measured on the axis of the shock tube, while the incident wave is measured at the top and bottom of the photograph, outside the acoustic shadow of the cylinder. The important features of the waves and volumes are indicated schematically in figure 18, and conventions for labelling the data on the $x-t$ diagrams are also given. The use of a series of still photos from several runs at different times is not a very precise method for measuring velocity, because of the variability from run to run of (i) the speed of sound inside and outside the gas volumes due to varying contamination and (ii) the strength of the incident shock wave due to the mechanical method used to generate shocks. However, in these experiments obtaining the superior spatial resolution of spark shadowgraphs over that of high-speed motion pictures was of higher priority than the precision in timing afforded by the latter. 
(a)

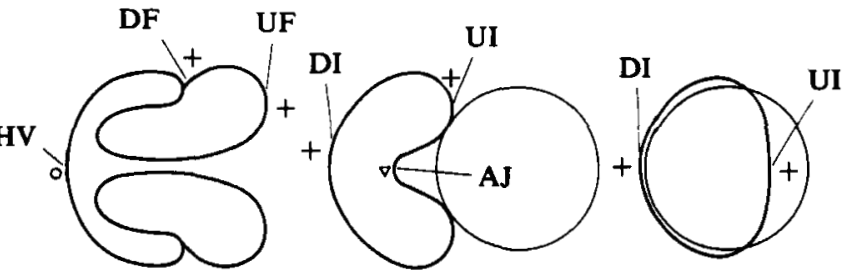

(b)

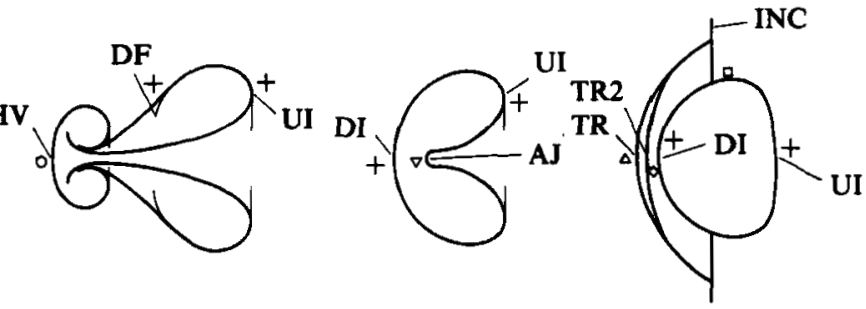

(c)

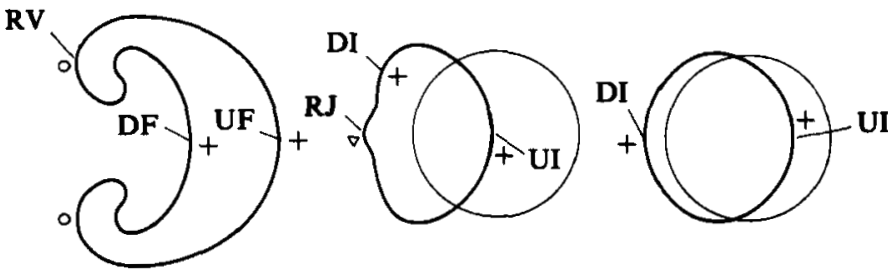

(d)

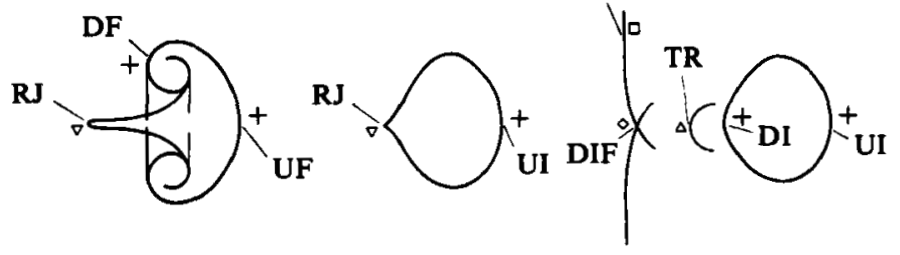

Froure 18. Schematic diagrams of the deformation histories of: $(a)$ helium cylinders; $(b)$ helium spheres; (c) R22 cylinders; (d) R22 spheres. UI, upstream interface (small time); DI, downstream interface (small time); UF, upstream interface (large time); DF, downstream interface (large time); AJ, air jet head; HV, helium vortex head; RJ, R22 jet head; RV, R22 vortex head; INC, incident and diffracted shock (outside of the shadow); TR, transmitted shock; TR2, secondary transmitted shock; DIF, crossing of the two branches of the diffracted shock.

\subsection{Divergent case}

The $x-t$ diagram of the interaction of a shock wave with a helium sphere is shown in figure 19. Velocities and times obtained from the figure, and from the $x-t$ diagram for cylindrical volumes, are given in table 2 . In order to assess, for example, the effects of gas contamination, it is useful to compare the observed velocities with those calculated from one-dimensional gasdynamics, as in table 1 (cf. table 3). Indeed, the relatively low observed values of the refracted wave velocity in the $M_{\mathrm{s}}=1.25$ case, namely, $960 v s .1140 \mathrm{~m} / \mathrm{s}$ in the one-dimensional interaction, suggests that contamination by air of the helium inside the volumes is significant. Using the shock strength of the refracted wave given by the pressure measurements $(\$ 5)$, we estimate that the speed of sound inside the cylinder is actually about $910 \mathrm{~m} / \mathrm{s}$ for the weak-shock case and $833 \mathrm{~m} / \mathrm{s}$ for the strong one $v s .1010 \mathrm{~m} / \mathrm{s}$ for pure helium. The 

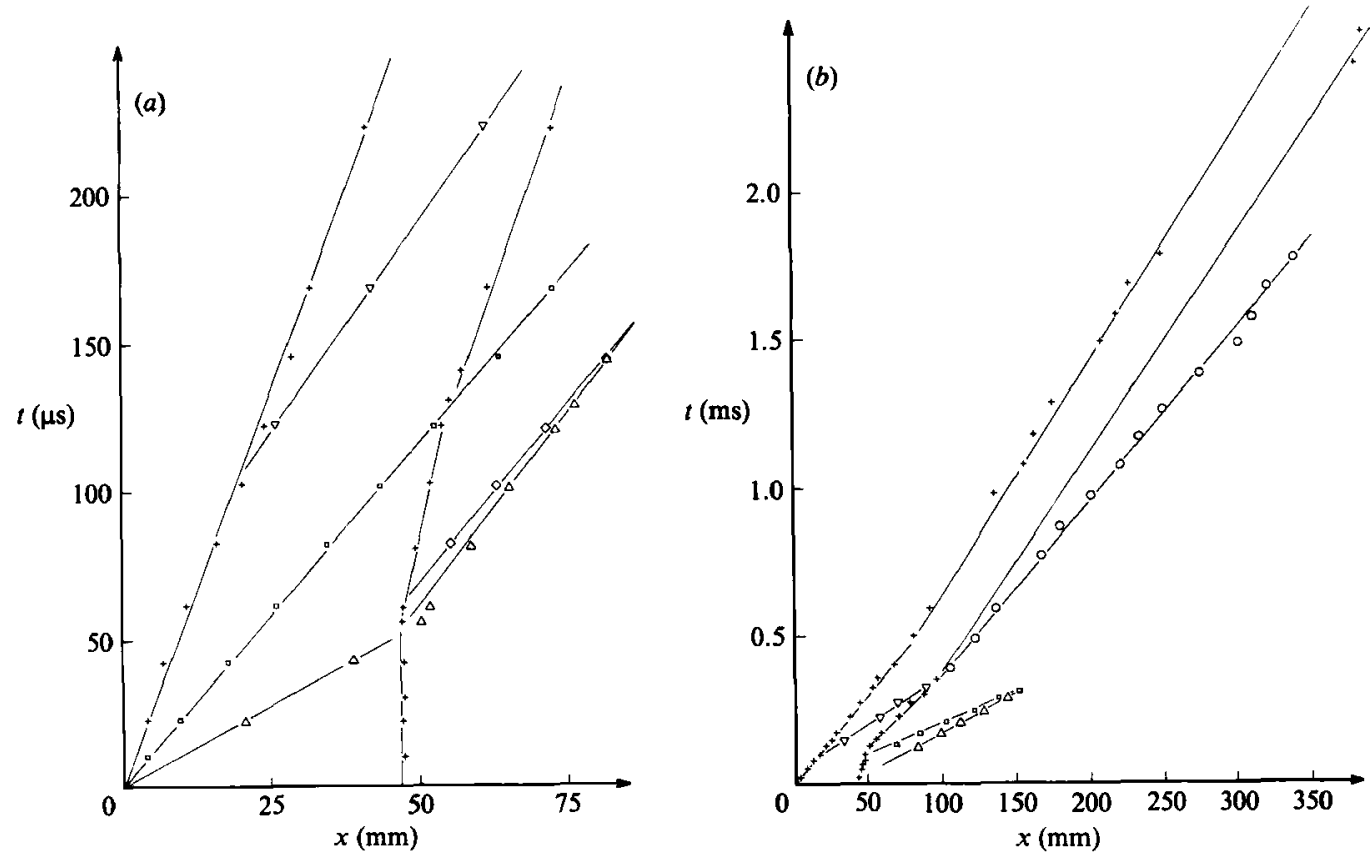

FIQURE 19. $x-t$ diagram of the interaction of an $M_{\mathrm{s}}=1.25$ shock wave with a helium sphere;

(a) small time, $(b)$ large time. Symbols are given in figure 18.

\begin{tabular}{|c|c|c|c|c|c|c|c|c|c|c|c|}
\hline$M_{\mathrm{s}}$ & $V_{\mathrm{s}}$ & $V_{\mathrm{R}}$ & $V_{\mathrm{T}}$ & $V_{\mathbf{u}_{i}}$ & $V_{\mathrm{uf}}$ & $V_{d_{1}}$ & $V_{\mathrm{df}}$ & $V_{\mathrm{j}}$ & $V_{\mathrm{v}}$ & $t_{\mathrm{j}}$ & $t_{\mathrm{v}}$ \\
\hline \multicolumn{12}{|l|}{ Sphere } \\
\hline 1.05 & 370 & - & - & 53 & 29 & 39 & 26 & 83 & 44 & 0.45 & 1.30 \\
\hline 1.10 & 380 & - & - & 87 & 50 & 67 & 50 & 140 & 75 & 0.20 & 0.70 \\
\hline 1.25 & 420 & 960 & 365 & 190 & 125 & $145^{a}$ & $165^{b}$ & 335 & 165 & 0.10 & 0.30 \\
\hline \multicolumn{12}{|c|}{ Cylinder } \\
\hline 1.085 & 400 & $950^{c}$ & 358 & 89 & $43^{d}$ & 69 & - & $125^{b}$ & 60 & 0.25 & 1.20 \\
\hline 1.22 & 410 & 900 & 393 & 170 & 113 & 145 & 97 & 230 & 128 & 0.10 & 0.70 \\
\hline
\end{tabular}

Velocities in $\mathrm{m} / \mathrm{s}$, times in $\mathrm{ms}$, estimated error of velocities: $10 \%$ except where noted.

$a$ Average of $105 \mathrm{~m} / \mathrm{s}$ for $t<t_{1}$ and $185 \mathrm{~m} / \mathrm{s}$ for $t<t_{\mathrm{v}}$ (cf. figure $19 a$ ) otherwise error $=30 \%$.

${ }^{b}$ Error $=30 \%$.

${ }^{c}$ Error $=20 \%$.

$d$ Error $=15 \%$.

$V_{s}$, velocity of incident and diffracted shocks (outside of the shadow). $V_{R}$, velocity of refracted shock. $V_{\mathrm{T}}$, velocity of transmitted shock. $V_{\mathrm{ul}}$, initial upstream interface velocity. $V_{\mathrm{uf}}$, final upstream interface velocity. $V_{\mathrm{di}}$, initial downstream interface velocity. $V_{\mathrm{df}}$, final downstream gas interface velocity. $V_{\mathrm{j}}$, velocity of jet head. $V_{\mathrm{v}}$, velocity of vortex pair or ring. $t_{\mathrm{j}}$, time to formation of the air jet. $t_{\mathrm{v}}$, time to formation of the vortex.

TABLE 2. Measured velocities for the helium volumes

mean value, $872 \mathrm{~m} / \mathrm{s}$, corresponds to a helium-air mixture with a mass concentration of air of 0.28 and a density $32 \%$ above the helium density. Henceforth we make a first-order correction for contamination by retaining these properties in our calculations. Contamination by helium of the air surrounding the cylinder also occurs. It is most apparent in the relatively large incident wave speed observed for the case 


\begin{tabular}{cccccrrrr}
\hline$M_{\text {s }}$ & $V_{\mathrm{s}}$ & $V_{\mathrm{R} 1}$ & $V_{\text {T1 }}$ & $V_{\text {u11 }}$ & $V_{\text {u1f }}$ & $V_{\text {d1 }}$ & $V_{2}$ \\
1.05 & 361 & 1037 & 358 & 40 & 30 & 23 & 28 \\
1.10 & 378 & 1063 & 372 & 78 & 59 & 45 & 54.7 \\
1.25 & 430 & 1140 & 413 & 184 & 138 & 105 & 129 \\
1.085 & 373 & 1055 & 368 & 67 & 50 & 38.5 & 46.8 \\
1.22 & 420 & 1125 & 405 & 164 & 123 & 94 & 114.8
\end{tabular}

Velocities in $\mathrm{m} / \mathrm{s}$.

$M_{\mathrm{s}}, V_{\mathrm{s}}$, input shock conditions. $V_{\mathrm{R} 1}$, velocity of wave transmitted from first interface. $V_{\mathrm{T} 1}$, velocity of wave transmitted from second interface. $V_{\mathrm{u} 11}$, initial upstream interface velocity. $V_{\mathrm{ul}}$, upstream interface velocity after return of the first internal wave reflected from the downstream interface. $V_{\mathrm{d} 1}$, downstream interface velocity. $V_{2}$, fluid velocity behind the incident shock.

\section{TABLE 3. One-dimensional interaction with air-helium interfaces}
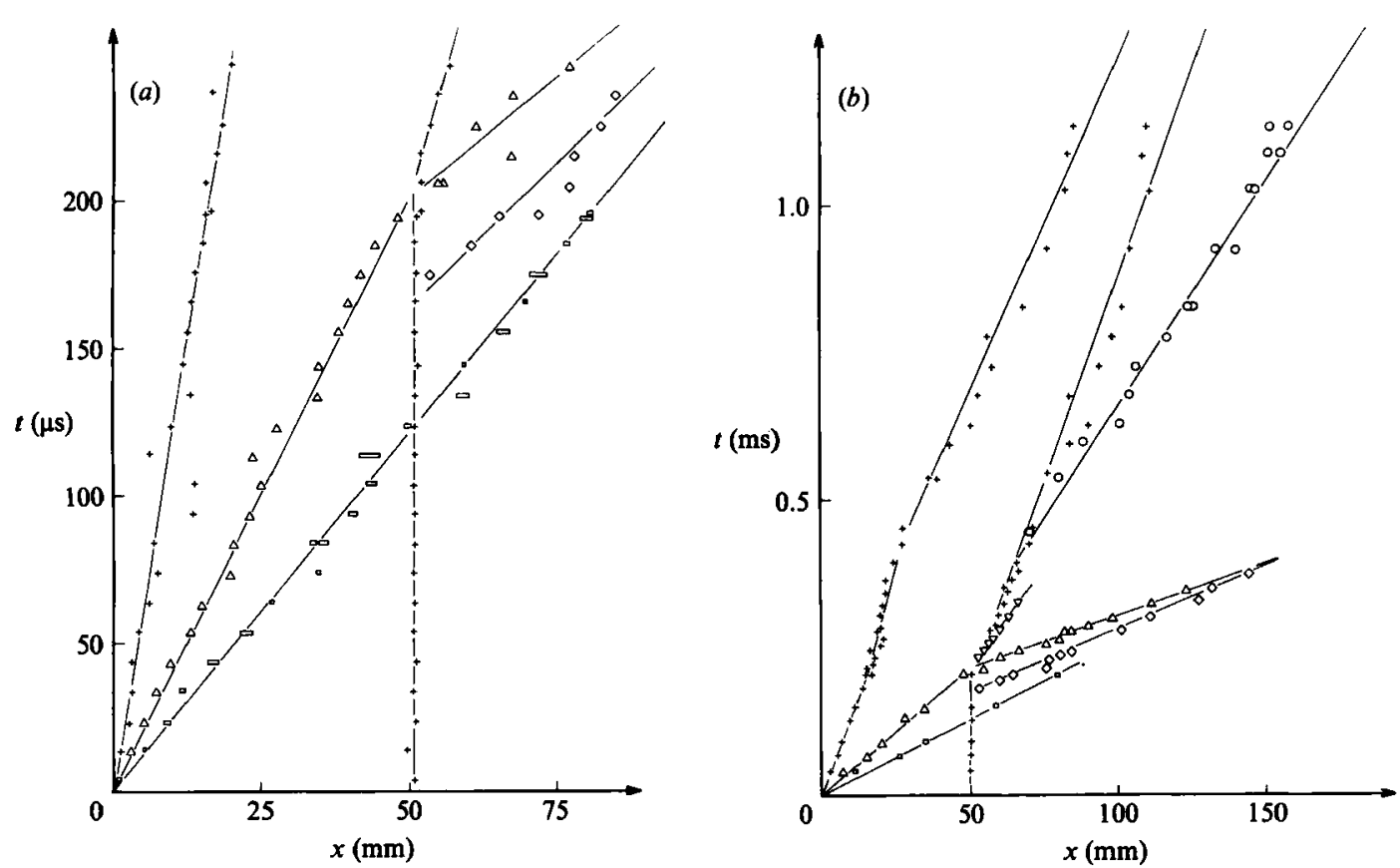

Figure 20. $x-t$ diagram of the interaction of an $M_{\mathrm{s}}=1.22$ shock wave with a R22 cylinder; (a) small time, (b) large time. Symbols are given in figure 18.

$M_{\mathrm{s}}=1.05$, which corresponds to a speed of sound of $369 \mathrm{~m} / \mathrm{s}$ and a mass concentration of helium in air of 0.02 . As might be expected this is a much lower contamination than inside the sphere, and is small enough that in subsequent calculations we take the ambient air to be pure, with sound speed $344 \mathrm{~m} / \mathrm{s}$.

The measurements show that the upstream interface initially acquires a velocity slightly higher than expected from one-dimensional gasdynamics, but, by the time the jet forms, its velocity is much larger. The downstream interface is also faster than predicted by one-dimensional gasdynamics. The jet that is seen to penetrate the helium volume in these experiments is analogous to the so-called spike of the Rayleigh-Taylor instability at a perturbed gaseous interface. After the formation of the vortex ring from the jet, the upstream and downstream faces of the original 
$\begin{array}{lllllllllllll} & M_{\mathrm{s}} & V_{\mathrm{s}} & V_{\mathrm{R}} & V_{\mathrm{T}} & V_{\mathrm{ui}} & V_{\mathrm{uf}} & V_{\mathrm{d}_{\mathrm{i}}} & V_{\mathrm{df}} & V_{\mathrm{j}} & V_{\mathrm{v}} & t_{\mathrm{v}} \\ \text { Sphere } & & & & & & & & \end{array}$

\begin{tabular}{|c|c|c|c|c|c|c|c|c|c|c|}
\hline 1.07 & 372 & 222 & 440 & 18 & 24 & 27 & 27 & $75^{\alpha}$ & - & 1.0 \\
\hline 1.25 & 421 & 239 & 574 & $60^{b}$ & 99 & 83 & 100 & $165^{a}$ & - & 0.4 \\
\hline 1.03 & 364 & 184 & 447 & - & - & - & - & - & - & - \\
\hline 1.085 & 382 & 220 & 510 & 42 & 35 & 35 & 35 & - & 54 & 1.0 \\
\hline 1.22 & 415 & 240 & 540 & $73^{c}$ & 90 & 78 & 78 & 153 & $130^{d}$ & 0.4 \\
\hline
\end{tabular}

a At late times (figure 14) the slender jet slows down to the velocity of the surrounding fluid.

b Error $=30 \%$.

c Error $=15 \%$.

d Weighted average of the two straight-line segments seen in figure $20 ; 81 \mathrm{~m} / \mathrm{s}$ for $t<0.2 \mathrm{~ms}$, $53 \mathrm{~m} / \mathrm{s}$ for $0.2<t<0.4 \mathrm{~ms}$, otherwise error $=35 \%$.

Notation defined in table 2.

TABLE 4. Measured velocities for the R22 volumes

$\begin{array}{lccccrcc}M_{\mathrm{s}} & V_{\mathrm{s}} & V_{\mathrm{R} 1} & \mathrm{~V}_{\mathrm{T} 1} & V_{\mathrm{u} 1_{1}} & V_{\mathrm{ulf}} & V_{\mathrm{d} 1} & V_{2} \\ 1.07 & 368 & 199 & 367 & 30 & 37 & 36.7 & 38.8 \\ 1.25 & 430 & 244 & 425 & 99 & 123 & 121.5 & 129 \\ 1.03 & 354 & 190 & 354 & 13 & 16 & 16.3 & 16.9 \\ 1.085 & 373 & 203 & 372 & 36 & 45 & 44.5 & 46.8 \\ 1.22 & 420 & 236 & 415 & 88 & 109 & 108.5 & 114.8\end{array}$

Notation defined in table 3.

TABLE 5. One-dimensional interaction with air-R22 interfaces

volume drift with small velocity relative to the surrounding gas, i.e. at velocity relative to the laboratory of about $V_{2}$, but the vortex moves noticeably faster.

\subsection{Convergent case}

The $x-t$ diagram for shock interaction with R22-filled cylinders is shown in figure 20. The velocities measured from the $x-t$ diagrams for both spheres and cylinders are shown in table 4 , and results from one-dimensional gasdynamics in table 5 . The initial velocities $V_{u_{i}}$ and $V_{d_{i}}$ of the upstream and downstream faces of the R22 volumes are lower than those predicted by the one-dimensional model, $V_{\mathrm{u}_{\mathbf{i}}}$ and $V_{\mathrm{d} 1}$, which are themselves smaller than $V_{2}$. In the cylindrical case the velocity of the line vortex pair which develops at later time is somewhat larger than $V_{2}$, in spite of the fact that vortices of the sense generated in this configuration should convect upstream relative to the surrounding fluid. We speculate that the contrary observation is due to blockage effects of the large and growing vortical structures. In the spherical case the vortical part of the structure at later times is slower than $V_{2}$.

Contamination in the R22-filled cylinder was estimated by calculating the speed of sound inside the cylinder from the average velocity of the refracted wave and its measured strength, as with the helium volumes. The resulting sound speed of, at most, $190 \mathrm{~m} / \mathrm{s}, 4 \%$ higher than the speed of sound in pure R22 $(182 \mathrm{~m} / \mathrm{s})$, suggests contamination by air of no more than $3.4 \%$ by mass. Similarly, estimates of the 
(a)
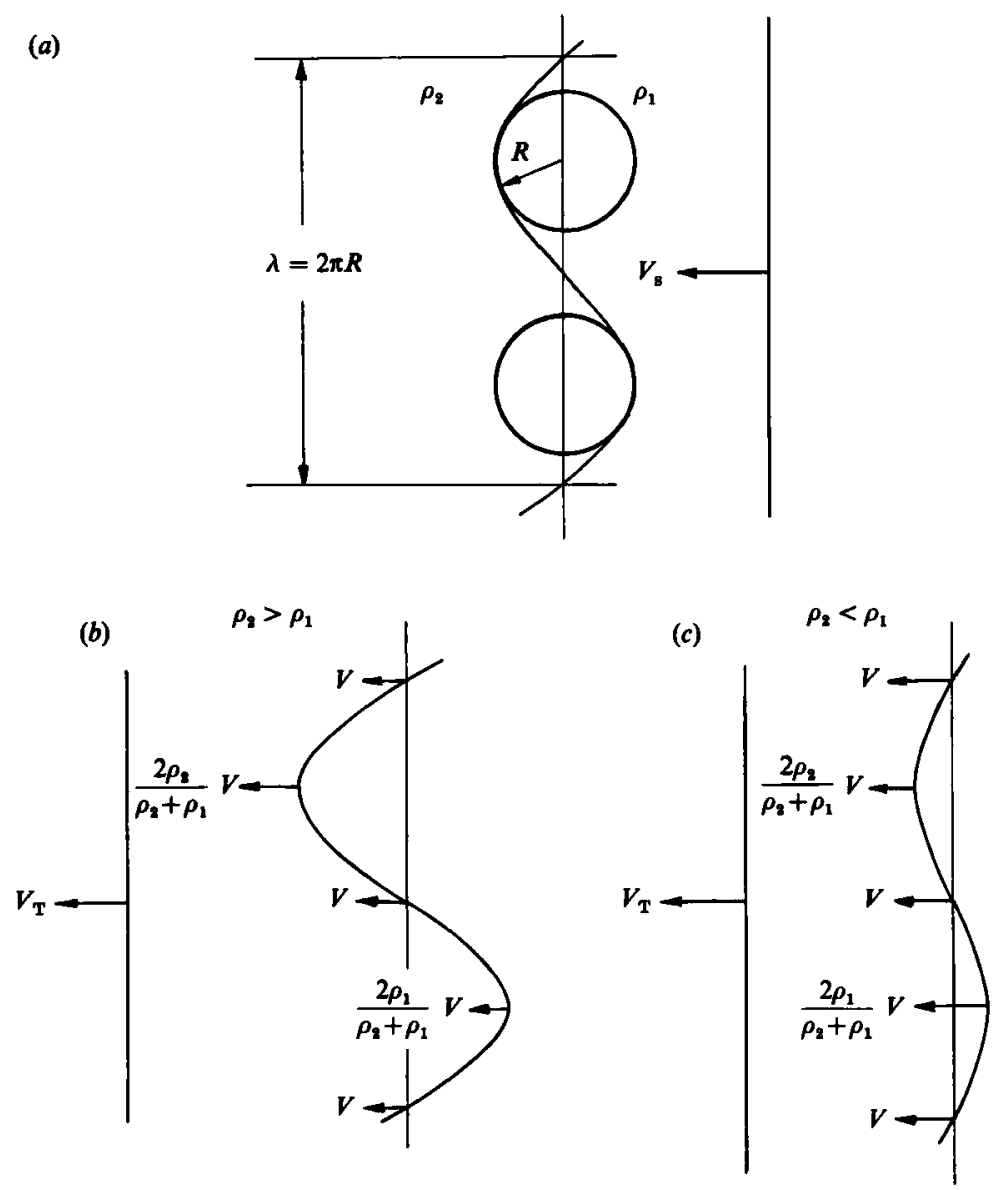

FigURE 21. Schematic representation of the Richtmyer-Meshkov instability of a sinusoidal interface; $(a)$ before interaction, matching of the cylinder and the sinusoid, $(b)$ after interaction, positive Atwood number, $(c)$ after interaction, negative Atwood number.

contamination of the air surrounding the R22 volumes from the speed of sound indicate only low levels of contamination. Thus, in all our calculations we neglect contamination of and by R22.

\section{Comparison of theory to experiment}

In this section we compare the measured deformation rates documented in $\$ 6$ with (i) the growth rate of small sinusoidal perturbations due to the impulsive acceleration of a plane interface predicted by Rayleigh-Taylor theory, (ii) a model of bubble acceleration and vortex generation proposed by Rudinger \& Somers (1960) and (iii) a simple calculation of the vorticity generated taking the jet to be a piston vortex generator. Mention is made of recent mathematical and numerical modelling by Picone \& Boris (1985, 1986) and Picone et al. (1986). It is instructive to investigate the connection mentioned in $\$ 1.2$ between shock-generated distortion of gas volumes and the Rayleigh-Taylor instability of plane interfaces because, if a cylinder, say, is taken to be representative of a sinusoidal perturbation of amplitude $\eta_{0}=R$, where $R$ is the radius of the cylinder, and wavelength $\lambda=2 \pi R$ (wavenumber $k=1 / R$ ), such 


\begin{tabular}{|c|c|c|c|c|}
\hline \multirow[b]{2}{*}{$M_{\mathrm{s}}$} & \multirow[b]{2}{*}{$V$} & \multirow{2}{*}{$\begin{array}{c}\text { Theory } \\
\mathscr{V}\end{array}$} & \multicolumn{2}{|c|}{ Experiment } \\
\hline & & & $\mathscr{V}_{\mathrm{ui}}$ & $\mathscr{r}_{j}$ \\
\hline \multicolumn{5}{|l|}{ Sphere } \\
\hline 1.05 & 37.9 & 1.876 & 1.40 & 2.19 \\
\hline 1.10 & 75.1 & 1.784 & 1.16 & 1.86 \\
\hline 1.25 & 177 & 1.432 & 1.07 & 1.89 \\
\hline \multicolumn{5}{|c|}{ Cylinder } \\
\hline 1.085 & 64.3 & 1.573 & 1.38 & 1.94 \\
\hline 1.22 & 158 & 1.432 & 1.08 & 1.46 \\
\hline
\end{tabular}

$V(\mathrm{~m} / \mathrm{s})$ calculated by one-dimensional theory $\left(V_{u_{1}}\right.$ of table 3$)$, corrected for contamination effects. $\mathscr{V}_{\mathrm{ui}}=V_{\mathrm{ui}} / V \cdot \mathscr{V}_{\mathrm{j}}=V_{\mathrm{j}} / V$.

TABLE 6. Upstream interface velocities of the helium volumes

$\dagger$ Corrected for contamination

\begin{tabular}{|c|c|c|c|c|c|}
\hline$M_{\mathrm{s}}$ & $M_{R} \dagger$ & $V_{R} \dagger$ & $\boldsymbol{V}$ & $\begin{array}{c}\text { Theory } \\
\mathscr{V}\end{array}$ & $\begin{array}{l}\operatorname{Ex}_{1} \\
\mathscr{V}_{d i}\end{array}$ \\
\hline \multicolumn{6}{|l|}{ Sphere } \\
\hline 1.05 & 1.029 & 897 & 23.9 & 1.953 & 1.6 \\
\hline 1.10 & 1.058 & 922 & 47.1 & 1.929 & 1.4 \\
\hline 1.25 & 1.143 & 996 & 110.9 & 1.869 & 1.3 \\
\hline \multicolumn{6}{|c|}{ Cylinder } \\
\hline 1.085 & 1.05 & 915 & 40.3 & 1.661 & 1. \\
\hline 1.22 & 1.126 & 982 & 98.8 & 1.622 & 1.4 \\
\hline
\end{tabular}

$V(\mathrm{~m} / \mathrm{s})$ calculated by one-dimensional theory $\left(V_{\mathrm{d} 1}\right.$ of table 3$)$, corrected for contamination effects. $\mathscr{V}_{\mathrm{d}_{1}}=V_{\mathrm{d}_{1}} / V$.

TABLE 7. Downstream interface velocities of the helium volumes

that the sine wave is tangential to and has the same curvature at crests and troughs as the cylinder (figure $21 a$ ), then the amplitude of the effective perturbation is very large $\left(k \eta_{0}=1\right)$, and deviations of the observations from linear theory may give some indication of the effects of finite amplitude and nonlinearity. For the sphere the wavenumber $k=\left(k_{x}^{2}+k_{y}^{2}\right)^{\frac{1}{2}}$ becomes $k=\sqrt{ } 2 / R$ and $k \eta_{0}=\sqrt{ } 2$.

\subsection{Shock-induced Rayleigh-Taylor instability}

When a shock wave of strength $M_{\mathrm{s}}$ impinges upon a plane interface distorted by small wavy perturbations of long wavelength, then according to the theory of the impulsive Rayleigh-Taylor instability (Markstein 1957a, $b$; Richtmyer 1960) the interface is given a mean translational velocity $V \equiv V_{u_{1}}$ (cf. tables 2 and 4) and a constant perturbation velocity $v$ at the troughs and crests,

$$
v= \pm \eta_{0} k V A
$$

The interface distorts at a constant rate and the velocity field persists because there is no further acceleration after shock impingement.

Transforming to the laboratory coordinate system, the velocity of the crests or troughs, normalized by the translational velocity $V, \mathscr{V}=1+v / V$, becomes

$$
\mathscr{V}=1 \pm \alpha A
$$




$$
\begin{aligned}
& \begin{array}{llcc} 
& & \text { Theory } & \operatorname{Exp} \\
M_{\mathbf{s}} & V & \mathscr{V} & \mathscr{V}_{\mathrm{ui}}
\end{array} \\
& \begin{array}{cccc}
\text { Sphere } & & & \\
1.07 & 30 & 0.333 & 0.60
\end{array} \\
& \begin{array}{llll}
1.25 & 99.2 & 0.442 & 0.60
\end{array} \\
& \text { Cylinder } \\
& \begin{array}{llll}
1.085 & 36.1 & 0.537 & 1.16
\end{array} \\
& \begin{array}{llll}
1.22 & 88.4 & 0.595 & 0.83
\end{array} \\
& V(\mathrm{~m} / \mathrm{s}) \text { calculated by one-dimensional theory }\left(V_{\mathrm{ui}_{1}} \text { of table } 5\right) . \mathscr{V}_{\mathrm{u}_{\mathrm{j}}}=V_{\mathrm{ul}} / V \text {. }
\end{aligned}
$$

TABLE 8. Upstream interface velocities of the R22 volumes

where the plus sign applies to a shock incident on a concave interface (trough) and $\alpha$ is 1 or $\sqrt{ } 2$ depending on whether the interface is locally cylindrical or spherical (figure $21 b, c$ ). Further, we adopt the first-order correction for nonlinearity suggested byRichtmyer (1960), accounting for the compression of the interface shape by the incident shock, by replacing $\eta_{0}$ with $\eta_{0}\left(1-V / V_{s}\right)$. In the present experiments the compression $1-V / V_{\mathrm{s}}$ varies between 0.59 and 0.90 for the helium experiments and from 0.77 to 0.92 in the $\mathrm{R} 22$ experiments.

\subsubsection{Helium: upstream interface}

In table 6 we compare the observed normalized initial interface velocity $\mathscr{V}_{u_{1}}$ and the subsequent normalized jet velocity $\mathscr{V}_{j}$ (cf. table 2 ) with the prediction $\mathscr{V}$ of linear stability theory. The observed initial rate of distortion is substantially smaller than that predicted for linear instability, even with the correction for compression, while the jet velocity is somewhat larger. While the normalized upstream interface velocities are similar in the spherical and cylindrical cases, the normalized jet velocity is slightly higher in the axisymmetric configuration. Both experiment and theory show that the rates decrease substantially with increasing wave strength.

\subsubsection{Helium: downstream interface}

As demonstrated by the growth of small-scale corrugations on the downstream side of the helium cylinder and sphere (e.g. figures $7 g$ and $8 c$ ) the downstream interface is initially destabilized by the shock that has already interacted with the upstream interface. In table 7 the observed normalized initial interface velocity $\mathscr{V}_{d_{1}}$ (cf. table 2 ) is compared with the prediction $\mathscr{V}$ of linear stability theory. Though the observed distortions on the sphere are again smaller than calculated by linear stability theory, even with the correction for compression, the agreement in the cylindrical case is substantially better, perhaps fortuitously. Again, there is little difference between the results in two dimensions and in three dimensions.

\subsubsection{R22: upstream interface}

In table 8 the observed normalized initial interface velocity $\mathscr{V}_{u_{i}}$ (table 4 ) is compared with the prediction $\mathscr{V}$ of linear stability theory. In this case the measured interface velocities are much larger than predicted, implying that the rate of distortion of the interface is again much less than in linear instability. We do not treat the behaviour of the downstream interface in the convergent case because the refracted shock focuses just at the interface, creating a situation very different from the interaction of a plane shock with an interface. 


\begin{tabular}{cccccccc} 
& & \multicolumn{4}{c}{$\mathscr{V}_{\mathrm{b}}$} & & \multicolumn{2}{c}{$\mathscr{V}_{\mathrm{v}}$} \\
\cline { 5 - 6 } \cline { 6 - 8 }$M_{\mathrm{s}}$ & $V_{2}$ & R-S & R-T & $\operatorname{Exp}$ & & R-S & $\operatorname{Exp}$ \\
Sphere & & & & & & \\
1.05 & 28 & 2.199 & 2.184 & 1.64 & & 1.523 & 1.57 \\
1.10 & 54.7 & 2.199 & 2.210 & 1.41 & & 1.523 & 1.37 \\
1.25 & 129 & 2.199 & 2.208 & 1.30 & & 1.523 & 1.28 \\
Cylinder & & & & & & \\
1.085 & 46.8 & 1.692 & 1.891 & 1.69 & & 1.140 & 1.28 \\
1.22 & 114.8 & 1.692 & 1.892 & 1.37 & & 1.140 & 1.12
\end{tabular}

R-S, Rudinger-Somers model. R-T, Rayleigh-Taylor model. $\sigma=\mathbf{0 . 1 8 2}$ for contaminated helium. $V_{2}$ in $\mathrm{m} / \mathrm{s}$. All other velocities normalized with $V_{2}$.

TABLE 9. Velocities of the helium volumes

\subsection{Bubble acceleration and vortex formation}

Rudinger \& Somers (1960) proposed a simple two-step model of shock-bubble interaction in which during the initial transients the bubble accelerates as a solid body to velocity $V_{b}$, and during the final development it transforms into a vortex ring with velocity $V_{\mathrm{v}}$ by the mechanism of Taylor (1953). The moving, undeformed bubble after the first step plays the role of Taylor's 'dissolved' vortex-generating disk, so $V_{b}$ is taken as the velocity of the disk. Rudinger \& Somers set the ratio of bubble gas density $\rho_{2}$ to surrounding gas density $\rho_{1}$ to be $\sigma=\rho_{2} / \rho_{1}$. Equating for the first step the impulse per unit volume $I$ experienced by the bubble to that experienced by the surrounding air,

$$
I=\rho_{1} V_{2}=\rho_{2} V_{\mathrm{b}}+k \rho_{1}\left(V_{\mathrm{b}}-V_{2}\right),
$$

where $k$ is the apparent mass fraction $=0.5$ for a sphere and 1.0 for a cylinder, the initial non-dimensional velocity of the volume results,

$$
\mathscr{V}_{\mathrm{b}}=\frac{V_{b}}{V_{2}}=\frac{1+k}{\sigma+k} .
$$

The transformation of the bubble into a vortex implies a decrease of the relative velocity

$$
V_{\mathrm{v}}-V_{2}=\beta\left(V_{\mathrm{b}}-V_{2}\right) \text {. }
$$

Taylor (1953) showed that $\beta=\mathbf{0 . 4 3 6}$ for the spherical case, and Rudinger \& Somers (1960) calculate $\beta=0.203$ for the cylinder. Thus the non-dimensional vortex velocity is

$$
\mathscr{V}_{\mathrm{v}}=\frac{V_{\mathrm{v}}}{V_{2}}=1+\beta \frac{1-\sigma}{\sigma+k} .
$$

As a matter of interest, we bring forth from tables 5 to 8 the average of the upstream and downstream interface velocities calculated on the Rayleigh-Taylor theory (without the correction for nonlinearity), this time normalized with $V_{2}$, to compare with the bubble velocities $V_{\mathrm{b}}$ of the present section,

$$
\mathscr{V}_{\mathrm{RT}}=\frac{V_{\mathrm{u1}_{1}}+V_{\mathrm{d} 1}}{2 V_{2}}\left(1+\alpha \frac{1-\sigma}{1+\sigma}\right) .
$$




\begin{tabular}{|c|c|c|c|c|c|c|}
\hline \multirow[b]{2}{*}{$M_{\mathrm{s}}$} & \multirow[b]{2}{*}{$V_{2}$} & \multicolumn{3}{|c|}{$\mathscr{V}_{\mathrm{b}}$} & \multicolumn{2}{|c|}{$\mathscr{V}_{v}$} \\
\hline & & $\mathrm{R}-\mathrm{S}$ & $\mathrm{R}-\mathrm{T}$ & Exp & R-S & $\operatorname{Exp}$ \\
\hline \multicolumn{7}{|l|}{ Sphere } \\
\hline 1.07 & 38.8 & 0.416 & 0.236 & 0.58 & 0.745 & 0.66 \\
\hline 1.25 & 129 & 0.416 & 0.235 & 0.55 & 0.745 & 0.77 \\
\hline \multicolumn{7}{|c|}{ Cylinder } \\
\hline 1.085 & 46.8 & 0.487 & 0.419 & 0.82 & 0.896 & 1.15 \\
\hline 1.22 & 114.8 & 0.487 & 0.417 & 0.66 & 0.896 & 1.13 \\
\hline
\end{tabular}

R-S, Rudinger-Somers model. R-T, Rayleigh-Taylor model. $\sigma=3.106$ for R22.

TABLE 10. Velocities of the R22 inhomogeneities

For experimental values, we take for $V_{b}$ the average of the measured initial upstream and downstream interface velocities $V_{\mathrm{u}_{1}}$ and $V_{\mathrm{d}_{1}}$ from tables 2 and $4 . V_{\mathrm{v}}$ is taken from tables 2 and 4, except for the R22 sphere where, since the vortex ring cannot be seen, we take the average of the final upstream and downstream edge velocities $V_{u f}$ and $V_{\mathrm{d}_{\mathrm{f}}}$ from table 4.

\subsubsection{Helium volume}

The measured and calculated velocities of the helium volumes are compared in table 9. The Rudinger-Somers (R-S) and the uncorrected Rayleigh-Taylor $(\mathrm{R}-\mathrm{T})$ models predict bubble and vortex velocities in rough agreement with those observed, but both models fail to account for the decrease of the velocities with increasing shock strength. Additionally, the vortex velocities observed in the present work agree approximately with those measured by Rudinger \& Somers (1960) for cylinders, namely $\mathscr{V}_{\mathrm{v}} \approx 1.3$ for $M_{\mathrm{s}}=1.12$ and $\mathscr{V}_{\mathrm{v}} \approx 1.15$ for $M_{\mathrm{s}}=1.22$ and 1.26. However, most important, the flow visualization of the present experiments shows that the helium bubble does not transform into a single vortex ring, as had previously been expected, but, in fact, splits into at least two structures, the largest containing little vorticity, and the smallest being an energetic vortex ring propagating rapidly ahead of the main structure. Thus calculation of the properties of the vortex is best carried out with a piston vortex-generator model (cf. below).

\subsubsection{R22 volume}

The comparison between theory and experiment is made in table 10. In this case the agreement between the $R-S$ and $R-T$ models is not as good, and the experimentally observed velocities are generally larger than predicted. As already noted, in the experiments the R22 structure grows so large that it interacts strongly with the shock-tube walls and acquires an abnormally large velocity. The growth of heavy bubbles after shock interaction was already noted for $\mathrm{SF}_{6}$ bubbles by Rudinger \& Somers who called attention to the explanation by Turner (1957) in terms of the Rayleigh-Taylor instability of rotating flows.

\subsection{Helium sphere: vortex-generator model}

In this section we estimate the strength of the vortex generated by the penetration of the helium spheres by the air jets (spikes) induced by the Rayleigh-Taylor instability. We treat the head of the jet as a solid piston. The piston velocity is $U_{\mathrm{p}}=V_{\mathrm{j}}-V_{\mathrm{b}}$, where $V_{\mathrm{b}}$ is the initial bubble velocity (cf. tables 9 and 10), and the 


\begin{tabular}{|c|c|c|c|c|c|c|c|c|c|}
\hline \multirow[b]{2}{*}{$M_{\mathrm{s}}$} & \multicolumn{5}{|c|}{ Measured } & \multicolumn{4}{|c|}{ Calculated } \\
\hline & $U_{\mathrm{p}}$ & $L_{\mathrm{p}}$ & $U_{\mathrm{v}}$ & $R$ & $r$ & $\Gamma_{\mathrm{p}}$ & $\Gamma_{\mathrm{v}}$ & $\Gamma_{\mathrm{v}} / \Gamma_{\mathrm{p}}$ & Re \\
\hline 1.05 & 37 & 38 & 16 & 10 & 5 & 0.70 & 0.72 & 1.03 & $0.48 \times 10^{5}$ \\
\hline 1.10 & 63 & 35 & 21 & 12 & 5 & 1.10 & 1.08 & 0.98 & $0.77 \times 10^{5}$ \\
\hline 1.25 & 167 & 30 & 37 & 15 & 4 & 2.51 & 2.21 & 0.88 & $1.75 \times 10^{5}$ \\
\hline \multicolumn{10}{|c|}{ Length in $\mathrm{mm}$, velocity in $\mathrm{m} / \mathrm{s}$, circulation in $\mathrm{m}^{2} / \mathrm{s}$. } \\
\hline \multicolumn{10}{|c|}{ TABLE 11. Piston and vortex ring parameters } \\
\hline
\end{tabular}

piston travel is $L_{\mathrm{p}}$. Following Kulkarny (private communication 1977), Maxworthy (1977) and Didden (1979), who studied the generation of vortex-rings by the impulsive motion of a piston that ejects a cylindrical volume of fluid from a short chamber into the surroundings, we calculate the circulation associated with the piston motion and the circulation of the vortex. Comparing the two values of circulation measures the 'efficiency' of the effective piston generator. The circulation and Reynolds number associated with the piston are

$$
\Gamma_{\mathrm{p}}=\frac{1}{2} U_{\mathrm{p}} L_{\mathrm{p}}, \quad \operatorname{Re}=\frac{\Gamma_{\mathrm{p}}}{\nu},
$$

where $\nu$ is the kinematic viscosity of air. $\Gamma_{\mathrm{v}}$, the circulation about the vortex, is obtained from the Kelvin formula for the velocity of a vortex ring of radius $R$ and core radius $r$ small compared to $R$,

$$
U_{\mathrm{v}}=\frac{\Gamma_{\mathrm{v}}}{4 \pi R}\left[\log \left(\frac{8 R}{r}\right)-\frac{1}{4}\right] .
$$

From our pictures we estimate $U_{\mathrm{p}}, L_{\mathrm{p}}, R, r$ and $U_{\mathrm{v}}=V_{\mathrm{v}}-V_{2}$. As might be expected, the most difficult quantity to measure is the core radius; of the photographs presented in this paper only one, figure $8(g)$, suggests a well-defined core. Fortunately the result does not depend strongly on this quantity. In table 11 we compare the piston circulation and the vortex circulation. We conclude that a surprisingly large fraction of the jet-associated circulation is transferred to the vortex. The numerical simulations discussed below give a similar result. The observed vortex rings are turbulent. This is consistent with a Reynolds number of the order of $10^{5}$.

\subsection{Numerical simulations}

Picone \& Boris (1985, 1986) and Picone et al. (1986) have carried out numerical simulations of our experiments using a finite-difference solution of the conservation equations for inviscid flow (ef. Picone et al. 1984). Their plots of density and vorticity contours are strikingly similar to the photographs presented in this work, and the magnitude of their calculated flow velocities is generally in good agreement with our observations.

\section{Summary and conclusions}

A new method for fabricating cylindrical gas inhomogeneities has made it possible to observe wavefronts inside and outside the volumes and to document the deformation of the boundaries of the inhomogeneity and the roll-up of vortical structures. 
The microfilm membrane used to separate the two gases did not perturb the geometry of the wave pattern and the motion of the gas interface, but had an effect on the strength of the shock waves measured very close to the cylindrical wall. Better control of the gas composition and use of a high-resolution high-speed motion picture camera would improve the precision of these measurements. The diameter of the volumes studied in this work was a large fraction of the test-section width, so blockage effects were experienced. Waves reflected from the shock-tube sidewalls modified the flow field at intermediate times.

\subsection{Wave patterns}

In these experiments shock waves incident on the gas inhomogeneities are relatively weak, so acoustic-ray tracing exhibits the wave behaviour. Generally, the major effect of nonlinearity is the overtaking and merging of transmitted waves downstream of the interaction.

The interaction of a shock with a cylinder filled with helium generates a diverging transmitted wave running ahead of the diffracted wave. At large times the diffracted wave is nearly as strong as was the incident wave. In the interior, an internal reflected wave focuses and appears both upstream of the cylinder as a backscattered wave which follows the external reflected wave and a secondary transmitted wave (glory) running just behind the main transmitted wave.

The wave pattern is more spectacular in the case of the R22 cylinder. Both the internal diffracted wave and refracted wave have caustics and the transmitted wave focuses just behind the cylinder. The strength of the converging shock waves in the interior leads to some interesting deviations of the observations from the wave-front diagrams obtained for acoustic pulses. In the experiment, the internal diffracted wave is very thick and the reflected waves show a double-wave structure. Neither of these effects is understood. A focal hot spot is left behind the focus of the transmitted wave. The merging of the transmitted and diffracted waves downstream of the cylinder occurs at approximately the same distance behind the R22 cylinder as for the helium cylinder. Again, the diffracted wave is about as strong as was the incident wave. Thus with light gas inhomogeneities the weak transmitted wave appears ahead of the main disturbance, while with heavy-gas inhomogeneities the weak wave is behind the main shock front.

\subsection{Deformation of the gas volumes}

The observed initial rate of deformation of the upstream edge of a helium sphere or cylinder, namely, a flattening followed by the formation of a jet/spike toward the interior, is better described by the linear theory of the shock-induced RayleighTaylor instability than that of the downstream edge of the helium inhomogeneity or the upstream interface on the R22 volume, because in the latter cases the perturbation becomes larger with the ensuing motion while in the former it decreases.

The distortion of the helium cylinder at large times conforms to the expectation that shock interaction with a gas lighter than the surroundings develops into a pair of vortices which stay close to each other and which move faster than the ambient fluid. The behaviour of the R22 cylinder agrees qualitatively with the prediction that an inhomogeneity heavier than air becomes a pair of vortices which move slower than ambient. However, in contrast to the helium volumes, which do not grow in lateral extent, the R22 volumes spread laterally very rapidly. The spreading causes the vortices at the edge to interact with the top and bottom walls of the test-section, so 
their velocities do not conform to theoretical predictions. A narrow jetlet is generated by the transmitted wave at its focus on the downstream side of the R22 volumes, especially on the sphere owing to axial focusing.

The development of the helium sphere at large times includes the unexpected result that an energetic vortex ring splits off from the main structure and propagates away along the axis of symmetry. Evidently the air spike penetrating the helium sphere acts like a piston vortex generator. The circulation in the vortex ring is almost the same as the circulation associated with the motion of the 'piston'. Sometimes a second vortex ring was observed to develop at the downstream edge of the main structure. Contemporary numerical simulations of the flow confirm the observed differences between the two-dimensional and axisymmetric behaviour. No vortex ring can be recognized in the large structure obtained from the R22 sphere, but its velocity is consistent with the existence of a vortex ring.

Many of the phenomena described here but not yet explained could perhaps be better understood if examined with a wider range of incident-shock-wave strength and inhomogeneity gas density, and with an experimental set-up in which blockage effects are not so important.

This work was supported by the National Science Foundation under Grant Number MEA 81-20092.

\section{REFERENCES}

Abd-el-Fattah, A. M., Henderson, L. F. \& Lozzi, A. 1976 Precursor shock waves at a slow-fast gas interface. J. Fluid Mech. 76, 157-176.

Abd-el-Fattah, A. M. \& Henderson, L. F. $1978 a$ Shock waves at a fast slow interface. J. Fluid Mech. 86, 15-32.

Abd-el-Fattah, A. M. \& Henderson, L. F. $1978 b$ Shock waves at a slow fast gas interface. J. Fluid Mech. 89, 79-95.

Andronov, V. A., Bakhrakh, S. M., Mokhov, V. N., Nikiforov, V. V. \& Pevinitskit, A. V. 1979 Effect of turbulent mixing on the compression of laser targets. Sov. Phys., J. Exp. Theor. Phys. Lett. 29, 56-59.

Anyiwo, J. C. \& Bushnell, D. M. 1982 Turbulence amplification in shock-wave boundary-layer interaction. AIAA J. 20, 893-899.

BaKer, L. \& Freeman, J. R. 1981 Heuristic model of the nonlinear Rayleigh-Taylor instability. J. Appl. Phys. 52, 655-663.

BockнoFf, M. \& RAUCH, D. 1973 Visualization and piezoelectric recording of the ultrasonic field within shock-loaded head-and container-models. 1973 Ultrasonics Symposium Proceedings, IEEE Cat. 73 CHO 807-8SU.

BrILL, D. \& ÜBERALL, H. 1970 Transmitted waves in the diffraction of sound from liquid cylinders. J. Acoust. Soc. Am. 47, 1467-1469.

Brown, G. L. \& Roshko, A. 1974 On density effects and large structure in turbulent mixing layers. J. Fluid Mech. 64, 775-816.

Bryson, A. E. \& Gross, R.W. F. 1961 Diffraction of strong shocks by cones, cylinders and spheres. J. Fluid Mech. 10, 1-16.

Catherasoo, C. J. \& Sturtevant, B. 1983 Shock dynamics in non-uniform media. J. Fluid Mech. 127, 539-561.

Chu, B. T. \& Kovasznay, L. S. G. 1957 Non-linear interactions in a viscous heat-conducting compressible gas. J. Fluid Mech. 3, 494.

Davy, B. A. \& Blackstock, D. T. 1971 Measurements of the refraction and diffraction of a short $N$ wave by a gas-filled soap bubble. $J$. Acoust. Soc. Am. 49, 732-737.

Didden, N. 1979 On the formation of vortex rings: Rolling-up and production of circulation. $Z$. angew. Math. Phys. 30, 101-116. 
Frowcs Williams, J. E. \& Howe, M. S. 1973 On the possibility of turbulent thickening of weak shock waves. J. Fluid Mech. 58, 461-480.

Folds, D. L. 1971 Target strength of focused liquid-filled spherical reflectors. J. Acoust. Soc. Am. 49, 1596-1599.

Friedlander, F. G. 1958 Sound Pulses. Cambridge University Press.

JAHN, R. G. 1956 The refraction of shock waves at a gaseous interface. J. Fluid Mech. 1, 457-489.

Jon es, D. S. 1978 Acoustic tunneling. Proc R. Soc. Edinb. 81 A, 1-21.

KeLLER, J. B. 1955 Diffraction by a convex cylinder. URSI Mich. Symp. on Electro Magnetic Wave Propagation.

KeLler, J. B. 1958 A geometrical theory of Diffraction Proc. Sym. Appl. Maths. vol. 7, Calculus of Variations and its Applications. McGraw-Hill.

Markstein, G. H. 1957 a Flow disturbances induced near a slightly wavy contact surface, or flame front, traversed by a shock wave. J. Aero. Sci. 24, 238.

Markstein, G. H. $1957 b$ A shock tube study of flame front-pressure wave interactions. 6th Intl Symp. Comb. pp. 387-398. Reinhold.

Marston. P. L. \& KingsBury, D. L. 1981 Acoustic scattering from fluid spheres: diffraction and interference near the critical scattering angle. $J$. Acoust. Soc. Am. 70, 1488-1495.

Marston, P. L. \& LANGLey, D. S. 1983 Glory- and rainbow-enhanced acoustic back-scattering from fluid spheres: Models for diffracted axial focusing. J. Acoust. Soc. Am. 73, 1464-1475.

Maxworthy, T. 1977 Some experimental studies of vortex rings. J. Fluid Mech. 81, 465-495.

MeSHкov, Y. Y. 1970 Instability of a shock wave accelerated interface between two gases. NASA $T T$ F-13, 074.

Meyer, K. A. \& BlewetT, P. J. 1972 Numerical investigation of the stability of a shock accelerated interface between two fluids. Phys. Fluids 15, 753-759.

Neubauer, W. G. \& Dragonatte, L. R. 1970 Observation of waves radiated from circular cylinders caused by an incident pulse. J. Acoust. Soc. Am. 48, 1135-1149.

Picone, J. M. \& Boris, J. P. 1985 Shock interactions with Freon bubbles in air. Bull. Am. Phys. Soc. $30,1706$.

PICONe, J. M. \& BorIs, J. P. 1986 Vorticity generation by shock propagation through bubbles in air. NRL Memorandum Rep. 5884. Naval Research Laboratory, Washington, D.C.

Picone, J. M., Boris, J. P., Oran, E. S. \& Ahearne, R. 1986 Rotational motion generated by shock propagation through a nonuniform gas. Proc. 15th Intl Symp. on Shock Waves and Shock Tubes (ed. D. Bershader \& R. Hanson), pp. 523-529. Stanford University Press.

Picone, J. M., Oran, E. S., Boris, J. P. \& Youne, T. R. 1984 Theory of vorticity generation by shock wave and flame interactions. Dynamics of Shock Waves, Explosions and Detonations pp. 429-448. AIAA.

Pierce, A. D. 1981 Acoustics. MoGraw-Hill.

RIChTMYER, R. D. 1960 Taylor instability in shock acceleration of compressible fluids. Commun. Pure Appl Maths 23, 297-319.

RUDINGER, G. 1958 Shock wave and flame interactions. Combustion and Propulsion, Third AGARD Coll. London. Pergamon.

RUDINGER, G. \& Somers, L. 1960 Behaviour of small regions of different gases carried in accelerated gas flows. J. Fluid Mech. 7, 161-176.

Smith, J. A., Coles, D., Roshko, A. \& Prasad, A. J. 1967 A description of the Galcit 6-inch shock tube. GALCIT report FM-67-1, California Institute of Technology.

Sturtevant, B. \& Kulkakny, V. A. 1976 The focusing of weak shock waves. J. Fluid Mech. 73, 651-671.

TAYLOR, G. I. 1950 The instability of liquid surfaces when accelerated in a direction perpendicular to their plane. I. Proc. R. Soc. Lond. A 201, 192-196.

TAYLOR, G. I. 1953 Formation of a vortex ring by giving an impulse to a circular disk and then dissolving it away. J. Appl Phys. 24. 1, 104.

Turner, J. S. 1957 Buoyant vortex rings. Proc. R. Soc. Lond. A 239, 61.

VAN DE Hulst, H. C. 1957 Light Scattering by Small Particles. Wiley. 
Walters, J. K. \& Davidson, J. F. 1962 The initial motion of a gas bubble formed in an inviscid liquid. Part 1. The two-dimensional bubble. J. Fluid Mech. 12, 408-416.

Walters, J. K. \& Davidson, J. F. 1963 The initial motion of a gas bubble formed in an inviscid liquid. Part 2. The three-dimensional bubble and the toroidal bubble. J. Fluid Mech. 17, 321-336. 